\title{
La investigación universitaria en Publicidad: producción y temática de las tesis doctorales (1971-2001)
}

\author{
Maria Jesús MARTínez PESTAÑA \\ maria.j.m.pestana@gmail.com \\ Universidad Complutense de Madrid
}

Recibido: 31/01/2011

Aceptado: 1/02/2011

\section{RESUMEN}

En este trabajo presentamos la producción de tesis doctorales realizadas en las universidades españolas desde 1971 hasta el año 2001. Para ello, se han realizado diferentes niveles de evaluación aplicando técnicas bibliométricas. Los niveles de evaluación realizados son: producción anual, producción por universidades, producción por Facultades, producción por líneas de investigación y producción por idiomas. Las tesis doctorales objeto de nuestro estudio son de tema publicitario y aparecen en los catálogos de las respectivas universidades.

Palabras clave: investigación en publicidad, tesis doctorales en publicidad, técnicas bibliométricas aplicadas.

University research in advertising, production and theme of the doctoral theses

\begin{abstract}
The aim of this paper is the production of doctoral thesis done in Spanish universities from 1971 to 2001. To achieve this goal, different levels of evaluation following bibliometric techniques have been applied. The evaluations are as follow: yearly productivity, university productivity, faculty productivity, main fields of research, language of the thesis. The doctoral thesis included in our study are of advertising content. The references of the thesis appear in the catalogs of their own universities.
\end{abstract}

Key words: advertising research, doctoral thesis on advertising, applied bibliometric techniques.

\section{OBJETO Y METODOLOGÍA}

El objeto de este trabajo es evaluar la producción de tesis doctorales de temas publicitarios realizadas en nuestro país durante el período comprendido entre 1971 y 2001. En el año 1971 se crea la licenciatura de Publicidad en nuestra universidad, de ahí la fecha de inicio de nuestro estudio. Además de la producción global de tesis doctorales, estamos interesados en evaluar también la producción por universidades, por Facultades, por líneas de investigación, y, finalmente, por las lenguas en que se han 
escrito los diferentes trabajos. Para obtener estos datos, hemos procedido a realizar diferentes niveles de evaluación, cuya metodología explicamos abajo.

En primer lugar, hemos procedido a identificar las tesis doctorales objeto de nuestro estudio durante el período establecido. Ya que nuestra intención era realizar diferentes evaluaciones, hemos aplicado técnicas procedentes de la bibliometría. Para ello, hemos creado una base de datos ad hoc en la que hemos introducido una serie de campos específicos de acuerdo con las evaluaciones que deseábamos realizar. La base de datos es de tipo relacional.

\section{INTRODUCCIÓN}

Una de las herramientas más útiles a la hora de evaluar el estado de la investigación proviene de las tesis doctorales ${ }^{1}$. Aunque consideradas como literatura gris no abundan este tipo de trabajos dada la escasez de difusión que sufren las tesis doctorales ${ }^{2}$. Sin embargo, creemos que es importe conocer, identificar y evaluar la investigación en el entorno de la universidad española en las tesis doctorales sobre publicidad ${ }^{3}$.

Para el estudio de la producción de tesis doctorales, interesa conocer en primer lugar la producción total de tesis doctorales en materia publicitaria, independientemente de la facultad o universidad en que haya tenido lugar la lectura y defensa de la tesis. Por ello, hemos creado, en primer lugar, una relación de autores que hayan presentado una tesis doctoral sobre publicidad, desde el año de inicio investigador en la universidad hasta el $2001^{4}$.

Para ello hemos trabajado con la información de treinta y una universidades cuya información sobre tesis doctorales ha sido actualizada recientemente ${ }^{5}$ en sus catálogos.

Con el objeto de manejar tal variedad de información hemos creado una base de datos relacional. Los datos han sido insertados en campos dentro de una tabla abierta y de estructura relacional.

${ }^{1}$ Véase el interesante estudio de López López, Pedro (1996): "La investigación bibliométrica en España (tesis doctorales)", Revista Española de Documentación Científica, 19 (1): 84-89.

${ }^{2}$ Villagrá ya recogió las restricciones que sufren las tesis doctorales en los estudios de producción científica en el área de ciencias sociales, por ser éstos un tipo de documentos que no se publica. Véase Villagrá Rubio, A. (1992): "Scientific production of Spanish universities in the fields of social sciences and languages", Scientometrics, 24 (1): 3-19.

${ }^{3}$ En su día publicamos un artículo sobre este tema. Las universidades actualmente están haciendo un esfuerzo para divulgar información sobre las tesis doctorales y ha repercutido en una mayor abundancia de datos sobre las tesis. De ahí que, aunque los resultados de este trabajo y el anterior sean parejos, trabajamos aquí con mayor volumen de información.

${ }^{4}$ Habíamos publicado anteriormente un artículo sobre el tema. Martínez Pestaña, María Jesús (2004): "La producción de tesis doctorales sobre temas publicitarios", Documentación de las Ciencias de la Información, 27: 237-267. Anotamos que este artículo ha sido revisado posteriormente, dadas las actualizaciones que han llevado a cabo las diferentes universidades.

${ }^{5}$ En enero del 2011 consultamos nuevamente los catálogos de las diferentes universidades, ya que la mayoría de ellas habían actualizado sus catálogos con nuevas entradas de tesis doctorales. 
Los campos insertados en nuestra base de datos de tesis doctorales son los siguientes:

1. Nombre completo del autor de la tesis doctoral.

2. Título completo de la tesis doctoral.

3. Año de lectura y defensa de la tesis doctoral.

4. Universidad en la que tuvo lugar la lectura y defensa de la tesis doctoral. Este campo y el siguiente permitirán que evaluemos a las universidades y Facultades en materia de tesis doctorales de la materia que nos ocupa.

5. Facultad en la que se ha leído la tesis doctoral. Nos interesaba aquí comprobar en qué Facultades ajenas a la comunicación o publicidad se han defendido estas tesis.

6. Tema o temas principales sobre los que versa la tesis doctoral. Este campo es de sumo interés ya que posibilita conocer las áreas de investigación más difundidas. Para ello, hemos asignado descriptores temáticos.

7. Idioma en el que se ha escrito y defendido la tesis doctoral. Además del castellano, comprobaremos si se usan otras lenguas españolas.

Con objeto de reunir toda esta información, hemos consultado las siguientes fuentes:

1. Base de datos de tesis doctorales TESEO ${ }^{6}$, Esta base de datos especializada en tesis doctorales está gestionada actualmente por el Ministerio de Educación. La fecha de inicio es 1976. No incorpora de forma regular el nombre del director hasta el año 1991. Así, echamos de menos la falta de exhaustividad y control hace tiempo, cuando comprobamos que no estaban incluidas las tesis doctorales de Vallina Velilla (1981) y Garrido Arilla (1982), por ejemplo, ni tampoco las de las universidades de la Fundación Universitaria San Pablo CEU. También detectamos errores en el campo de los años para alguna de las tesis que hemos estudiado.

2. Catálogos bibliográficos de la Biblioteca Nacional Española ${ }^{7}$.

3. Catálogos de la Facultad de Ciencias de la Información de la Universidad Complutense de Madrid ${ }^{8}$.

4. Publicaciones del Consejo de Universidades? .

5. Portal de la Biblioteca Virtual Miguel de Cervantes. Permite que, a través del buscador de tesis doctorales, se realice la búsqueda por autores, títulos, o universidades. Como novedad ofrece una relación de 48 universidades

\footnotetext{
${ }^{6} \mathrm{http} / / /$ www.educacion.es/educacion/universidades/educacion-superior-universitaria/tesis-doctorales-teseo.html

${ }^{7}$ Se puede consultar a través de: www.bne.es.

${ }^{8}$ Facultad de Ciencias de la Información, Universidad Complutense de Madrid (1983):”Tesis doctorales (1978-1982)", en Bibliografia de las Ciencias de la Información, Madrid: Facultad de Ciencias de la Información, Universidad Complutense de Madrid, pp. 86-87.

${ }^{9}$ Consejo de Universidades, Secretaría General (1990),op. cit.
} 
españolas y, además, alguna extranjera. Sigue la clasificación de materias de la UNESCO, así como la CDU ${ }^{10}$.

6. Servicios de bibliotecas de los portales de las diferentes universidades. Aunque, no son exhaustivas en el apartado de las tesis doctorales, son la única herramienta con la que contamos los investigadores. La Base de datos CISNE, de la Universidad Complutense de Madrid ${ }^{11}$, así como la web de la Universidad Autónoma de Barcelona ${ }^{12}$ han sido las más utilizadas en nuestra investigación. Destacamos además las correspondientes a la Universidad Autónoma de Madrid ${ }^{13}$, Universidad de Alicante $^{14}$, Universidad de Comillas ${ }^{15}$, Universidad de Deusto ${ }^{16}$, Universidad de Granada $^{17}$, Universidad de La Laguna ${ }^{18}$, Universidad de Las Palmas ${ }^{19}$, Universidad de Málaga ${ }^{20}$, Universidad de Murcia $^{21}$, Universidad de Navarra $^{22}$, Universidad de Oviedo ${ }^{23}$, Universidad de Salamanca ${ }^{24}$, Universidad de Sevilla ${ }^{25}$,Universidad de Valencia ${ }^{26}$, Universidad de Valladolid $^{27}$,Universidad de Zaragoza ${ }^{28}$, Universidad del País Vasco ${ }^{29}$, Universidad Jaume I de Castellón ${ }^{30}$, Universidad Politécnica de Cataluña ${ }^{31}$, Universidad Politécnica de Valencia ${ }^{32}$, Universidad Ramón Llull ${ }^{33}$ y, finalmente, la Fundación Universitaria San Pablo-CEU ${ }^{34}$

7. Base de datos REBIUN ${ }^{35}$ (Red Española de Bibliotecas Universitarias) es de sumo interés ya que se trata de un catálogo colectivo de acceso a las bibliotecas de las universidades españolas, siendo por ello de gran utilidad.

\footnotetext{
${ }^{10}$ Véase: www.cervantesvirtual.com.

${ }^{11}$ http://cisne.sim.ucm.es/

12 http://www.uab.es/

${ }^{13} \mathrm{http}: / /$ www.uam.es/

14 http://www.ua.es/

${ }^{15} \mathrm{http}: / / \mathrm{www}$. upcomillas.es

$16 \mathrm{http}: / / \mathrm{www}$. deusto.es/

${ }^{17}$ http:// www.ugr.es/

18 http://www.ull.es/

${ }^{19} \mathrm{http}: / /$ www.ulpgc.es/

${ }^{20} \mathrm{http}: / /$ www.uma.es/

${ }^{21}$ http://www.um.es/

${ }^{22} \mathrm{http}: / /$ www.unav.es/

${ }^{23} \mathrm{http}$ ://www.uniovi.es/

24 http://www.usal.es/

${ }^{25} \mathrm{http}$ ://www.us.es/

${ }^{26} \mathrm{http}: / / w w w . u v . e s /$

${ }^{27} \mathrm{http}: / / w w w . u v a . e s /$

${ }^{28}$ http://www.unizar.es/

${ }^{29} \mathrm{http}: / / w w w . e h u . e s$

${ }^{30} \mathrm{http}$ ://www.uji.es/

31 http://www.upc.es/

32 http://www.upv.es/

${ }^{33} \mathrm{http}: / / w w w . u r l . e s /$

${ }^{34} \mathrm{http}: / / w w w . c e u . e s /$

$35 \mathrm{http}$ ://www.rebiun.org
} 
De hecho, creada en 1988, a iniciativa de los directores de bibliotecas de las universidades españolas, tiene como objeto implementar los niveles de servicios de estas bibliotecas.

8. Portal Dialnet ${ }^{36}$ de la Universidad de la Rioja es, sin duda, la más completa en todos los datos que acompañan a las tesis.

9. Tesis doctorales en red (TDR) ${ }^{37}$ es de gran utilidad especialmente para las tesis del área de Cataluña, aunque incluye otras universidades.

10. Buscadores Kriptia ${ }^{38}$ y Mastesis ${ }^{39}$. De reciente creación, ambos permiten la búsqueda temática.

En definitiva, todos los buscadores se nutren de la información facilitada por las universidades. Y los errores y las ausencias pasan de un lugar a otro. Sería deseable que las mismas universidades revisasen sus catálogos para corregir errores e incluir toda la información, como la fecha de lectura y defensa de la tesis, y la nómina completa del tribunal y director de la tesis.

\section{EVALUACIÓN DE LA PRODUCCIÓN ANUAL}

Desde el año 1971 hasta el 2001 hemos identificado un total de 165 tesis doctorales ${ }^{40}$. Sin embargo, no se leyó la primera tesis sobre publicidad hasta el año 1974, que fue presentada por José Ramón Sánchez Guzmán en la Facultad de Ciencias Económicas y Empresariales de la Universidad Complutense de Madrid y no como consideró erróneamente Méndiz Noguero ${ }^{41}$ en el año 1976.

Además del tardío inicio, también la investigación en tesis doctorales sobre publicidad es tímida en los primeros años. Así volveremos a encontrar una sola tesis doctoral en los años 1976 y 1979, a pesar de la excepción que anuncia el año 1975 en el que se registran 2 tesis, aunque aún en Facultades diferentes de la de Ciencias de la Información, ya que se presentan en la Facultad de Derecho y de Ciencias Económicas y Empresariales de la Universidad Complutense.

La producción de estos primeros es casi irrelevante, si no fuese porque se presentan las primeras tesis doctorales sobre publicidad en la universidad española. A la tesis doctoral de Sánchez Guzmán (1974), le siguen García Nieto-Serrano (1975), López Guerra (1975) y Martín Serrano (1976). En el año 1979 veremos la primera tesis

${ }^{36} \mathrm{http}: / / \mathrm{www}$.dialnet.unirioja.es/

${ }^{37} \mathrm{http}: / /$ www.tdr.cesca.es/

${ }^{38} \mathrm{http}: / /$ www.kriptia.com/

${ }^{39} \mathrm{http}: / / \mathrm{www} . \mathrm{mastesis.com} /$

${ }^{40}$ Véase la Relación de Tesis doctorales en el Anexo.

${ }^{41}$ Méndiz Noguero, Alfonso (2000): "Orígenes, evolución y desafíos actuales de la docencia publicitaria en España”, Comunicación y Sociedad, 13 (2), 118-225. 
doctoral en una Facultad de Ciencias de la Información, que fue presentada por Desantes Guanter ${ }^{42}$ en la Universidad de Navarra.

Aunque estos primeros representan una producción escasa o nula, del $0,6 \% \mathrm{y}$ del $1,2 \%$, son importantes porque anticipan la singladura futura de la investigación sobre publicidad. Es decir, en lugar de limitar la investigación sobre temas publicitarios a las Facultades de Ciencias de la Información y Comunicación manifiestan la tendencia a investigar sobre temas publicitarios en otras Facultades.

El año 1981 arranca con la fuerza de 4 tesis doctorales, 2 de ellas en Facultades de Ciencias de la Información, en la sección de publicidad. La primera de Pérez Tornero (Universidad Autónoma de Barcelona) y la segunda de Vallina Velilla (Universidad Complutense de Madrid). Volveremos a encontrar 4 tesis en el año 1985 y 3 tesis en el año 1986 , que representan un porcentaje del $2,4 \%$ y $1,8 \%$ respectivamente.

En contrapartida, los años 1982, 1984, 1987 y 1988 cuentan con sólo 2 tesis en cada caso, apenas por encima del $1 \%$.

En el año 1989 se produce un crecimiento importante, ya que se presentan 7 tesis en ese año, que vienen a representar un $4,2 \%$ sobre el total. Es más, con la salvedad del año siguiente (1990) en el que se presentan 2 tesis (1,2\% sobre el total), el año 1989 inicia lo que va ser el asentamiento de la investigación en el área de publicidad en el seno de la universidad, como apreciaremos.

Así pues, desde 1991 hasta el año 2001 tiene lugar lo que denominaremos la consolidación de la investigación universitaria en el área de publicidad. Esta fase de expansión se refleja en la lectura habitual de tesis doctorales sobre publicidad en diferentes universidades y Facultades. Tanto es así, que la productividad de esta etapa es del $80 \%$ sobre la totalidad. Numéricamente, queda expresado en que de las 165 tesis doctorales leídas desde 1971 hasta el año 2001, 132 tesis doctorales fueron presentadas en el período 1991 a 2001.

En la Tabla 1 hemos detallado los resultados de la producción anual. En la columna de la izquierda figura el año, en la central el número total de tesis defendidas sobre publicidad y en la de la derecha los porcentajes Tabla 1 (Número de tesis doctorales por años y porcentaje (\%) desde 1971-2001).

Con la excepción del año 1990 en el que aún encontramos 2 tesis, el año 1989 marca la tendencia positiva ascendente a lo largo de todo el período de estudio. A partir del año 1989 hasta el 1991 se aprecian crecimientos importantes. Si el año 1989 marcaba la tendencia alcista con 7 tesis doctorales, idéntica producción corresponde a los años 1992 y 1994, a pesar de que son los años de menor producción desde el año 1989, pero ya representan porcentajes significativos superiores al $4 \%$ en cada caso.

En general, a partir del año 1993 vamos a encontrar una producción anual superior a las 10 tesis, es decir, con valores superiores al $6 \%$ anual. Significativamente, los años 1988 y 2001 son los más productivos, con 22 y 20 tesis doctorales, respectivamente. Conjuntamente, representan más del $25 \%$ respecto a la producción total.

${ }^{42}$ Dada la escasa divulgación de las tesis doctorales en estos años, erróneamente creímos que la primera tesis en un Facultad de Ciencias de la Información databa del año 1981. Felizmente, hemos corregido esta información. 
Tras el análisis anual, vamos a proceder a considerar los niveles de producción por períodos extensos. Las tres décadas desde 1971 al año 2000 suman una producción conjunta de 145 tesis y un porcentaje respecto al conjunto de las 165 tesis del 87,86\%.

El año 2001 señala sin duda la tendencia positiva de los años precedentes, como anuncian las 20 tesis de este año y un porcentaje superior al $12 \%$

Gráficamente, podemos apreciar en la Tabla 2 (Número de tesis doctorales por período de años (1971-2000) y 2001) la evolución por décadas y la proyección que indica el año 2001.

No cabe duda que el incremento del número de tesis doctorales a partir del año 1991 es muy superior con creces a los años precedentes. Las 112 tesis presentadas y defendidas en el período desde el año 1991 hasta el 2000 así lo confirman.

Si prestamos atención a los porcentajes, el menor corresponde (3\%) a la década de 1971 a 1980 y el más elevado (67\%) el que corresponde al período de 1991 a 2000.

De hecho, este crecimiento importante a partir del año 1991 indica la estabilización de la actividad investigadora sobre materia publicitaria. Esta circunstancia es paralela a la estabilización de la actividad publicitaria empresarial en España, especialmente en el ámbito de las agencias publicitarias y empresas relacionadas con el sector.

Sin duda, la tendencia alcista que hemos observado en la última década encuentra la continuación positiva en el año 2001 y que, como prolegómeno del nuevo siglo, viene a ser positiva y continuadora de los años precedentes.

\section{EVALUACIÓN DE LA PRODUCCIÓN POR UNIVERSIDADES}

Tras observar cuáles han sido los niveles de producción anuales y la evolución de las diferentes épocas y la proyección del año 2001, vamos a proceder a analizar cómo se distribuyen las tesis doctorales entre las diferentes universidades españolas. Las 165 tesis doctorales estudiadas se distribuyen entre treinta y una universidades españolas.

Iniciaremos nuestro análisis por las universidades con mayores niveles productivos. Para una mejor comprensión, hemos decidido agrupar las universidades por índices de productividad en orden decreciente. Así, en un primer grupo (1) hemos incluido las universidades con más de 20 tesis doctorales; en el segundo grupo (2), aquellas universidades que cuentan entre 20 y 10 tesis doctorales; en el tercer grupo (3) aquellas que superan las 5 tesis y no pasan de 10; en el cuarto grupo (4) las que están por debajo de 5 tesis doctorales y por encima de 2 . En un último grupo (5) están incluidas las universidades que tan sólo cuentan con una tesis.

El nivel más elevado de producción corresponde al grupo (1) con una sola universidad. El grupo (2) está representado por dos universidades. Ambos grupos aportan más del 52\% de la producción global. El grupo (3), con seis universidades universidades, aporta casi la misma producción que el grupo (2). Similar situación presenta el grupo (4) que, aún agrupando a doce universidades posee una producción similar a las de los grupos (2) y (3). Finalmente, el grupo (5) cuenta con la producción única de una sola tesis doctoral de cada una de las diez universidades que incluyen este grupo. 
La Tabla 3 (Número total de tesis por niveles de productividad de universidades agrupadas) ilustra la producción de cada uno de los grupos. La columna de la izquierda refiere el grupo adscrito de acuerdo con los niveles de producción arriba indicados. La columna central presenta el total de tesis conjunto de cada grupo; y en la columna de la derecha aparecen indicados los volúmenes de producción acumulados.

Observamos que el grupo (1) es el más productivo con diferencia, mientras que los grupos (2) y (4) presentan una producción similar, superados ambos grupos por el grupo (3). En el nivel de producción más bajo figura el grupo (5).

Recordemos que fueron la Universidad Complutense, la Universidad Autónoma de Barcelona y la Universidad de Navarra las universidades que introdujeron las primeras Facultades de Ciencias de la Información en el año 1971, aunque sólo la Universidad Complutense de Madrid y la Universidad Autónoma de Barcelona integrarían la Licenciatura de Publicidad desde sus comienzos. Aunque con diferencias, son estas tres universidades las que se sitúan a la cabeza en volumen de tesis doctorales sobre temas publicitarios.

El nivel de producción más elevado lo presenta la Universidad Complutense de Madrid, que con 56 tesis doctorales acapara casi el 34\% de la producción de tesis doctorales. Ello justifica que figure en solitario en el grupo (1).

Le siguen a distancia agrupadas (2) la Universidad Autónoma de Barcelona y la Universidad de Navarra con una producción conjunta de 30 tesis doctorales y un porcentaje respecto al conjunto del $18 \%$. Este nivel de producción es semejante al que presenta el grupo (4) que agrupa, en orden descendente atendiendo a la producción, a la Universidad de La Laguna, la Universidad de Zaragoza, la Universidad del País Vasco, la Universidad de Salamanca, la Universidad Politécnica de Valencia, la Universidad Autónoma de Madrid, la Universidad Carlos III, la Universidad de Barcelona, la Universidad de Murcia, la Universidad Politécnica de Cataluña, la Universidad Ramón Llull y, finalmente, la Universidad San Pablo-CEU.

En nivel productivo conjunto, le siguen a las anteriores el grupo (3) con una producción conjunto de 38 tesis doctorales que, porcentualmente representa el $23 \%$. A la cabeza de este grupo se sitúa la Universidad de Sevilla, seguida de la Universidad de Valencia, la Universidad de Oviedo y, finalmente, la Universidad de Alicante, la Universidad de Granada y la Universidad de Málaga.

En el nivel más bajo se encuentran diez universidades que han aportado una tesis doctoral únicamente y cuya producción conjunta apenas supera el $6 \%$.

En la Tabla 4 (Número de tesis doctorales por universidades) figura en detalle la producción individual de cada universidad. En la columna de la izquierda figura el grupo que hemos asignado anteriormente. A continuación figura la universidad en cuestión. La producción individual para cada universidad figura en la columna siguiente donde aparece el año de defensa de la tesis; el número de tesis doctorales anuales aparece entre paréntesis. En la columna final de la derecha aparece el número total de tesis de cada universidad.Tras estudiar el nivel de producción de las universidades españolas en tesis doctorales de contenido publicitario, nos interesa también comprobar en qué Facultades han sido defendidas. 


\section{EVALUACIÓN DE LA PRODUCCIÓN POR FACULTADES}

Nos interesa comprobar el nivel de producción de las Facultades de Ciencias de la Información o Ciencias de la Comunicación en comparación con el resto de las Facultades. Además, prestaremos especial atención a las Facultades de Ciencias Económicas y Empresariales, debido al peso cada vez más importante que representan estas Facultades en este área de investigación. De hecho, hemos encontrado trece tipos de Facultades donde se han defendido tesis doctorales sobre publicidad, como indicamos en la Tabla 5 (Facultades donde se han defendido tesis de contenido publicitario).

Las Facultades de Ciencias de la Información y Comunicación concentran más de la mitad de la producción con 84 tesis, mientras que las Facultades de Ciencias Económicas y Empresariales acaparan el 20\% de la producción, con una producción conjunta de 33 tesis.

Es de destacar también el importante papel que juegan otras Facultades ajenas a las anteriores en la investigación sobre temas publicitarios. En especial, las Facultades de Derecho, Filología y Filosofía y Letras, cuya producción individual va desde las 9 tesis en el primer caso a las 8 tesis de las dos últimas; y representan individualmente un porcentaje cercano al $5 \%$.

También como dato curioso, las Facultades de Psicología, Ciencias Políticas e Ingeniería y Escuelas Técnicas han contribuido en el interés por la investigación de contenido publicitario, aunque sólo cuenten con 5 ó 4 tesis, es decir, algo más del 2\% respecto al conjunto.

También de interés son las Facultades de Bellas Artes, Educación y Medicina que, en su modesta contribución, han contribuido a la investigación sobre publicidad en las tesis, aunque no superen las 2 y 3 tesis. Como tampoco debemos olvidar las contribuciones de una Facultad de Farmacia y otra de Geografía e Historia, aunque sólo hayan aportado una única tesis cada una.

Pues bien, si las Facultades de Ciencias de la Información y Ciencias de la Comunicación concentran el grueso de la producción, es la Facultad de Ciencias de la Información de la Universidad Complutense de Madrid la que acapara la mayor concentración productiva con 41 tesis y un porcentaje cercano al $25 \%$. A ésta le siguen con una producción neta de 15 y 12 tesis las Facultades de Ciencias de la Información de la Universidad Autónoma de Barcelona y la de la Universidad de Navarra y que representan individualmente unos porcentajes del $9 \%$ y del $7 \%$ en cada caso. También debemos mencionar las contribuciones de las Facultades de la Universidad de La Laguna y la Universidad de Sevilla con 4 tesis en cada caso. Como también son de interés las aportaciones de 2 tesis en las Facultades de Información/Comunicación de las universidades de Málaga, del País Vasco, Ramón Llull y San Pablo-CEU.

Con respecto a las Facultades de Ciencias Económicas y Empresariales éstas presentan una mayor dispersión en su distribución en las universidades, ya que nos encontramos con dieciocho universidades con este tipo de facultad, frente a las nueve universidades presentes en las Facultades de Ciencias de la Información o Comunicación. Así, a la cabeza figuran las Facultades de Ciencias Económicas de la Universidad Complutense 
de Madrid, de la Universidad de Alicante y de la Universidad de Valencia con 5 y 4 tesis. A éstas hay que añadir las Facultades de la Universidad de Sevilla y de la Universidad de Zaragoza con 3 tesis cada una; y y de la Universidad de Granada aunque sólo cuenten con 2 tesis en cada caso. El resto de las Facultades de Ciencias Económicas y Empresariales presentes en el resto de las universidades contribuyen únicamente con una sola tesis, como puede observarse en la Tabla 6 (Facultades en que se han presentado tesis doctorales).

También debemos destacar la presencia de las Facultades de Derecho en la investigación en tesis doctorales de contenido publicitario, presente en las tesis de seis Facultades, situándose a la cabeza la facultad de la Universidad Complutense.

\section{Relación de Universidades:}

UA: Universidad de Alicante; UAB: Universidad Autónoma de Barcelona; UAM: Universidad Autónoma de Madrid; UB: Universidad de Barcelona; UC: Universidad de Comillas; UCA: Universidad de Cantabria; UCIII: Universidad Carlos III; UCM: Universidad Complutense de Madrid; UD: Universidad de Deusto; UG: Universidad de Granada; UJC: Universidad Jaume I de Castellón; ULL: Universidad de La Laguna; ULP: Universidad de Las Palmas; UMA: Universidad de Málaga; UMU: Universidad de Murcia; UN: Universidad de Navarra; UNED: Universidad Nacional de Educación a Distancia; UO: Universidad de Oviedo; UPC: Universidad Politécnica de Cataluña; UPM: Universidad Politécnica de Madrid; UPV: Universidad del País Vasco; UPVA: Universidad Politécnica de Valencia; URL: Universidad Ramón Llull; US: Universidad de Sevilla; USA: Universidad de Salamanca;.USC: Universidad de Santiago de Compostela; USP: San Pablo-CEU; UV: Universidad de Valencia; UVL: Universidad de Valladolid; UZ: Universidad de Zaragoza.

\section{Fuente: Elaboración propia}

Las Facultades de Filología están presentes en cinco universidades, situándose a la cabeza la de la Universidad Complutense, la de la Universidad de Oviedo y la de la Universidad de Valencia. Con idéntica producción las Facultades de Filosofía y Letras presentan una mayor dispersión representadas en ocho universidades y una sola tesis.

Las Facultades de Psicología, de Ciencias Políticas y de Ingeniería y Escuelas Técnicas Superiores figuran con una producción pareja, aunque a la cabeza se sitúa la facultad de la Universidad Complutense en el primer caso, la de la Universidad Carlos III en el segundo y la de la Universidad Politécnica de Cataluña en el tercero.

Con una producción menor, señalemos las Facultades de Bellas Artes y de Educación, seguidas por la Facultad de Medicina de la Universidad de Sevilla.

Finalmente, en solitario, la Facultad de Farmacia de la Universidad de Granada y la Facultad de Geografía e Historia de la Universidad de Oviedo han contribuido con una investigación en tesis doctoral de contenido publicitario.

Tras estudiar la producción anual y su evolución, la producción atendiendo a las universidades y a las Facultades en que se han defendido tesis doctorales de contenido 
publicitario, creemos que es importe conocer los contenidos preferentes de los investigadores.

\section{EVALUACIÓN DE LA PRODUCCIÓN POR LÍNEAS DE INVESTIGACIÓN}

Los estudios sobre líneas de investigación permiten conocer cuáles de ellas son más o menos activas, su vigencia y antigüedad. A cada una de las tesis doctorales estudiadas hemos asignado un contenido o contenidos, en función de la línea investigadora. El hallazgo de unas líneas de investigación comunes, nos ha permitido evaluar los intereses investigadores preferentes, como podemos observar en la Tabla 7 (Líneas de investigación presentes en las tesis doctorales).

Por ello, hemos ordenado las líneas de investigación preferentes en orden decreciente, comenzando por las líneas de investigación comunes a varios trabajos. En la columna central figuran los autores y entre paréntesis el año de defensa de la tesis doctoral. En la columna de la derecha, hemos anotado el número de tesis doctorales orientadas en una misma línea de investigación.

La investigación centrada en los medios publicitarios se sitúa a la cabeza como línea preferente entre los investigadores, representando el $12 \%$ respecto al conjunto. Se inicia hacia la mitad de 1980 y son la Universidad de Granada, con la tesis de González Cachinero (1984) y la Universidad Politécnica de Valencia con la tesis de Saborit (1986) las que inician esta línea investigadora. A pesar de que la Universidad Complutense concentra el grueso de tesis sobre esta línea, ésta está presente en prácticamente todas las universidades. Aunque el interés ha recaído sobre los medios audiovisuales y prensa, destaquemos el interés por el medio interactivo e Internet (Salas Nestares, Sádaba Chalezquer, Díaz Soloaga), así como el cine como medio publicitario (Rivero, Amiguet).

Tanto la línea sobre campañas publicitarias como la orientada hacia el consumidor y el impacto de la publicidad cuentan con igual número de tesis que superan un porcentaje del $10 \%$ en cada caso. La investigación sobre campañas publicitarias goza de más antigüedad ya que se inicia en 1975 (López Guerra) en la Universidad Complutense, aunque se interrumpe hasta 1989 (Sánchez Medrano). Aunque la temática es amplia, destaquemos la coincidencia de tres tesis sobre publicidad bancaria (Herrero Olaizola, Zaldumbide y Bocigas). También es una línea ampliamente desarrollada en diferentes universidades.

La línea de investigación sobre el consumidor y el impacto de la publicidad es una línea de investigación más moderna, ya que la primera aparece en 1988 en la Universidad Autónoma de Barcelona (Soler Pujals) para desarrollarse con profusión en diferentes universidades. La temática es variada, como lo prueban, por ejemplo, la orientada hacia el consumidor infantil (Núñez Gómez) o el impacto negativo de la publicidad (Rodríguez Rodríguez, Carrillo Durán).

El mensaje publicitario cuenta también con un gran desarrollo y está presente en una variedad de universidades, abarcando un porcentaje del 9\%. Encontramos la primera en 
la Universidad de Alicante en 1982 (Feliu Garcia), y continuará con fuerza a partir de 1989 (Eguizábal).

La investigación dedicada a la eficacia publicitaria goza también de un amplio espectro representativo y acapara más del 8\% respecto al conjunto. Se inicia en 1985 en la Universidad Complutense (León Sáez de Ybarra), aunque su desarrollo principal tiene lugar a partir de 1995. Se da la circunstancia que, a excepción de las presentadas en las Facultades de Ciencias de la Información y Comunicación la gran parte han sido presentadas en Facultades de Ciencias Económicas y Empresariales.

En contrapartida a las áreas anteriores, la investigación sobre el uso de elementos simbólicos en publicidad cuenta ya con una tradición, puesto que la primera data de 1982 (Garrido Arilla) y fue presentada en la Universidad Complutense de Madrid. El repertorio investigador sobre el uso de elementos simbólicos en publicidad ha sido muy diverso, como prueban las dirigidas hacia el confucionismo (Kim), la ecología (Olivares), la lengua (Sábate), o el uso de elementos femeninos y/o masculinos (R.Soto, Rey, P. Ruíz, Garrido), por mencionar algunas.

La investigación sobre empresas de publicidad es la línea de investigación que goza de más antigüedad, y acapara más del 6\%. Se inicia en 1974 (Sánchez Guzmán) en la Universidad Complutense de Madrid, aunque no vuelve a continuar hasta el año 1985 en la Universidad Complutense (Fernando Martín) y la Universidad Autónoma de Barcelona (Gozálvez Serrano). Los temas aquí son diversos, como son los dedicados a la agencia de publicidad (Visiedo, Giquel, Río Pérez), la empresa de relaciones públicas (Arceo, G. Nieto), centrales de compras de medios (Pérez Latre), empresas de producción (Pérez Bianco), o el centro de documentación publicitaria (Martín Martín).

El área dedicado a la legislación es también una de las líneas de investigación que disfruta de más antigüedad, ya que la encontramos en 1979 en la Facultad de Ciencias de la Información de la Universidad de Navarra (Desantes). Esta línea de investigación, aunque abarca un $6 \%$, tiene una trayectoria particular, ya que, a pesar de ser una línea temprana, no volverá a aparecer hasta 1987 en la Universidad Autónoma de Barcelona (Madrenas i Boadas).

También la línea de investigación dedicada a la gestión publicitaria, con un porcentaje superior al 5\%, arranca tempranamente, ya que aparece en 1976 en la Universidad de Sevilla (Martín Armario). Sin embargo, tendremos que esperar hasta el año 1992, para encontrar la siguiente (Leal López) en la misma universidad.

La creatividad y el discurso disfrutan de igual atención por parte de los investigadores, ya que representan el $4,8 \%$ en cada caso. Con respecto a la creatividad, ésta sin duda es una línea de investigación tardía, ya que aparece en 1991 en la Universidad Autónoma de Barcelona (Ricarte Bescós) y cuenta con un gran impulso en esta universidad (Ramiro, Roca Correa). Como también cuenta con un gran impulso en la Universidad Complutense (Marcos Molano, Baños González, Roca Correa).

La línea orientada hacia el discurso goza de una trayectoria más temprana, ya que la encontramos en 1984 en la Universidad de Valencia (Puchol), y continuará después en la Universidad de Málaga (Sánchez Corral).

Aunque con menor representación, ya que sólo cuenta con algo más del 2,4\%, la investigación sobre planificación de medios es una línea de investigación moderna que 
se inicia en 1991 en el seno de la Universidad Complutense (Mazo del Castillo) y continuará en esta misma universidad (Díez Medrano).

A continuación, figuran cuatro líneas de investigación con idéntica representación y un porcentaje individual del $1,8 \%$. Se trata de las orientadas a la docencia, la función de la publicidad, la inversión publicitaria y las relaciones públicas. El área de investigación orientada a la docencia aparece en la Universidad de Valencia en 1981 (Beltrán), al igual que la dedicada a la función de la publicidad que también se inicia en 1981 pero en la Universidad de Barcelona (Siurana). Más tardíamente, la investigación sobre inversión publicitaria aparece en 1986 en la Universidad Politécnica de Madrid (Hernández Martínez). Finalmente, aunque la investigación sobre relaciones públicas se inicia tempranamente en 1975 en la Universidad Complutense (García-Nieto), no volverá a aparecer hasta 1990 en la Universidad Autónoma de Barcelona (Noguero).

Con una representación menor del 1,2\%, figuran las orientadas hacia la estandarización publicitaria, la estrategia y la semiótica. La estandarización publicitaria aparece en la Universidad de Murcia en 1999 (Flores) aplicada a las empresas exportadoras. La estrategia publicitaria cuenta con su primera tesis presentada en la Universidad Complutense en 1987 (Pérez González). Aunque la investigación dedicada a la semiótica aparece en 1981 en la Universidad Autónoma de Barcelona (Pérez Tornero), no volverá a aparecer hasta el año 1993 en la Universidad Complutense (Caro).

Finalmente, otras dos líneas de investigación, representadas con una sola tesis, ante la imposibilidad de asociarlas con otras líneas de investigación, hemos decidido mantenerlas en solitario, dado su nivel de especificidad. Se trata de la investigación sobre patrocinio y la teoría de sistemas. La primera arranca en 1994 en la Universidad de Navarra (Carrogio), mientras que la segunda data de 1981 y se inicia en la Universidad Complutense (Vallina).

Veamos ahora, a continuación, cuáles son los centros que han atraído y concentrado a la mayor parte de los investigadores en tesis doctorales.

\section{PRODUCCIÓN POR IDIOMAS}

Es bien sabido la importancia que tiene el idioma en que se ha escrito una investigación. Todo investigador es conocedor que si aporta nuevo conocimiento a su área de investigación el proceso de divulgación será más amplio si se ha escrito en una lengua común a otros centros de investigación. Sin duda, el idioma es un tema de importancia, puesto que puede favorecer o no la divulgación de un documento científico. Al mismo tiempo, ilustra el nivel de presencia investigadora de una lengua determinada.

La representación de otras lenguas en el apartado de tesis doctorales es menor como se puede apreciar en el resumen de la Tabla 8 (Distribución de lenguas en tesis doctorales (1971-2001)

En el área de tesis doctorales, todas se han defendido y escrito en castellano, con la excepción de 2 tesis. Una primera, de Ferrés i Prats, en catalán en el año 1989, fue 
presentada en la Universidad Autónoma de Barcelona. La segunda, también en catalán, aparece casi diez años más tarde. Su autor es Sabaté López y presenta su tesis sobre el uso del catalán en la publicidad. Sabaté la presentó en la Universidad Ramón Llull en 1998. Al año siguiente aparecerá una tercera en la Universidad Autónoma de Barcelona (Fernández Cavia).

Estas 3 tesis presentadas en catalán, dado la escasa representación respecto al resto (tal sólo un 1,81\%) las confirman como excepciones frente a las 160 tesis doctorales presentadas en castellano.

En el año 2001, en la Universidad de Navarra se presentan 2 tesis doctorales en inglés, de De Mooji y Kliatchko. Aunque la representación es pequeña, no deja de tener interés.

\section{CONCLUSIONES}

Nuestro estudio se ha basado en la muestra de 165 tesis doctorales de contenido publicitario defendidas en treinta y una universidades españolas, desde 1971 hasta el año 2001.

1. Los años con una producción más elevada corresponden al año 1998 con 22 tesis y 2001 con 20 tesis.

2. La década más productiva corresponde a los años 1991-2000 con 112 tesis y un porcentaje del $67 \%$.

3. El año 2001 mantiene la tendencia positiva de la década precedente.

4. La Universidad Complutense de Madrid presenta el nivel más alto de producción con una concentración de 56 tesis (34\%). A distancia seguidas por la Universidad Autónoma de Barcelona y la Universidad de Navarra, con 17 y 13 tesis, respectivamente.

5. Las Facultades de Ciencias de la Información y Comunicación concentran 84 tesis, más de la mitad de la producción. Las Facultades de Ciencias Económicas y Empresariales concentran 33 tesis (20\%).

6. Las líneas de investigación preferentes son el medio publicitario, presente en 20 tesis, campañas publicitarias y la orientada hacia el consumidor y el impacto de la publicidad, con 17 tesis en cada caso.

7. El castellano es la lengua en que se han leído la mayor parte de las tesis. En catalán se han leído 3 tesis y 2 en inglés. 


\section{ANEXO. RELACIÓN DE TESIS DOCTORALES}

ALDÁS MANZANO, JOAQUÍN (1997): Modelos de determinación de la cobertura y la distribución de contactos en la planificación de medios publicitarios impresos, Facultad de Ciencias Económicas y Empresariales, Universidad de Valencia.

ALVAREZ ROQUE, CRISTINA (1999): La publicidad en la prensa de santa cruz de Tenerife en la segunda mitad del siglo XIX. Facultad de Ciencias de la Información, Universidad de La Laguna.

ÁLVAREZ RUÍZ, XOSE ANTON (2001): La función social de la publicidad de la ONCE. Cómo las campañas de la ONCE han conseguido cambiar las imágenes dominantes sobre las personas discapacitadas y acelerar su integración social, Facultad de Ciencias de la Información, Universidad Complutense de Madrid.

AMAYA ROMAN, ANA M. (1991): Análisis de la publicidad medicamentosa en revistas pediátricas españolas. Facultad de Medicina, Universidad de Sevilla.

AMIGUET ESTEBAN, JOSÉ MANUEL (1999): El concepto de marca en la comunicación audiovisual y publicitaria del cine. Un estudio de los indicadores de marca en el caso Almodóvar, Facultad de Ciencias de la Información, Universidad Complutense de Madrid.

ANGELES VILLENA, JUAN DE LOS (1993): Valoración empresarial de la creatividad publicitaria, Facultad de Ciencias de la Información, Universidad de Navarra.

ARCEO VACAS, ALFREDO (1993): Estrategias de relaciones públicas: los planteamientos de las grandes agencias del sector, Facultad de Ciencias de la Información, Universidad Complutense de Madrid.

BACA LAGOS. VICENTE (1994): Las representaciones de los hombres y las mujeres en la televisión. Facultad de Ciencias de la Información, Universidad Complutense de Madrid.

BAÑOS GONZÁLEZ, MIGUEL (2000): Métodos heurísticos y creación publicitaria, Facultad de Ciencias de la Información, Universidad Complutense de Madrid.

BARRIO GARCÍA, SALVADOR DEL (1999): La publicidad comparativa como estrategia publicitaria: influencia de determinados elementos moderadores en su eficacia, Facultad de Ciencias Económicas y Empresariales, Universidad de Granada.

BELTRAN DE TENA, ROSARIO (1981): Propaganda y enseñanza. los aspectos formales de la propaganda y su posible aplicación a la tecnología didáctica. Facultad de Filosofía y Ciencias de la Educación, Universidad de Valencia.

BETÉS RODRÍGUEZ, ENRIQUETA (1999): La construcción del relato publicitario en el medio radio: Formatos, universo temático, personajes y estética sonora, Facultad de Ciencias de la Información, Universidad Complutense de Madrid.

BLASCO LÓPEZ, MARIA FRANCISCA (2000): Aprendizaje y marketing: Investigación experimental del juego de simulación como método de aprendizaje, Facultad de Ciencias de La Información, Universidad Complutense de Madrid.

BOCIGAS SOLAR, OLGA (1998): El cambio de orientación de los grandes bancos privados españoles analizado a través de la publicidad realizada en el periodo 1982- 
1994. Especial referencia al Banco Santander, Facultad de Ciencias Económicas y Empresariales, Universidad de Comillas.

BOHOYO DIAZ, EDUARDO (2000): Aproximación hermeneútica a las mitologías del anuncio televisivo. Facultad de Bellas Artes, Universidad Politécnica de Valencia.

BRINGUE SALA, JAVIER (1998): La comunicación publicitaria infantil en televisión. Facultad de Comunicación, Universidad de Navarra.

CALDERÓN MARTÍNEZ, PURIFICACIÓN (1999): Estimación de la cobertura y distribución de exposiciones en el medio televisión. Modelos de agregación e individuación, Facultad de Ciencias Económicas y Empresariales, Universidad de Alicante.

CARO ALMEDA, ANTONIO (1993): La publicidad de la significación (marco, concepto y taxonomía), Facultad de Ciencias de la Información, Universidad Complutense de Madrid.

CARRILLO DURÁN, MARÍA VICTORIA (2001): La influencia de la publicidad, entre otros factores sociales, en los trastornos de la conducta alimentaria: Anorexia y bulimia nerviosas, Facultad de Ciencias de la Información, Universidad Complutense de Madrid.

CARROGIO GUERÍN, MARCOS (1994): Patrocinio, deporte y olimpismo: Estrategias de comunicación, Facultad de Ciencias de la Información, Universidad de Navarra.

CASCANTE SERRATOSA, CARLOS (2001): El concepto de STAS (Short Term Advertising Strenght) de John Philip Jones como medida de la eficacia de la publicidad y sus repercusiones en la planificación de medios. Análisis de su aplicación en España a través de tres casos prácticos, Facultad de Ciencias de la Comunicación, Universidad Ramón Llull.

COMITRE NARVAEZ, ISABEL (2000): Traducción y publicidad. Aproximación a una estrategia traslativa (francés-español). Facultad de Filología, Universidad de Málaga.

CONEJERO LÓPEZ, MARTA (1993): Publicidad: Escenas de persuasión, Facultad de Filología, Universidad de Valencia.

CORREA GARCIA, RAMON IGNACIO (1996): La publicidad como discurso pedagógico. Facultad de Educación, Universidad Nacional de Educación a Distancia.

CORREDOIRA ALFONSO, LORETO (1989): Régimen jurídico del patrocinio en España y en la comunidad europea. Facultad de Ciencias de la Información, Universidad de Navarra.

DE MOOIJ, MARIEKE (2001): Convergence and divergence in consumer behavior. Consequences for global marketing and advertising. Facultad de Comunicación, Universidad de Navarra.

DESANTES GUANTER, JOSE MARIA (1979): Propaganda, publicidad y relaciones publicas en la legislación de las cajas de ahorros. Facultad de Ciencias de la Información, Universidad de Navarra.

DESCALZO GONZALEZ, ANTONIO (1997): Competencia leal e información veraz en un mercado eficiente. (Una aproximación a la publicidad comercial). Facultad de Ciencias Sociales y Jurídicas, Universidad Carlos III. 
DÍAZ SOLOAGA, PALOMA (2001): El proceso de construcción de imagen de marca en Internet: Una investigación aplicada a la creación de marcas en jóvenes, Facultad de Ciencias de la Información, Universidad Complutense de Madrid.

DIEZ ARROYO, M. LUISA (1995): La retorica del mensaje publicitario: Un tratamiento interdisciplinar. Facultad de Psicología, universidad de Oviedo.

DÍEZ MEDRANO, JAVIER (1997): Planificación publicitaria en prensa por las grandes entidades financieras: Un modelo de comunicación prospectivo, Facultad de Ciencias de la Información, Universidad Complutense de Madrid.

DURAN MARTINEZ, RAMIRO (2001): La lengua inglesa en la publicidad española: Una aproximación pragmática. Universidad de Salamanca, Facultad de Filología.

DOMÍNGUEZ ÁVILA, CARMEN LUCÍA (1994): Publicidad y valores. Modelos de consumidor y análisis de valores en la publicidad televisiva española (1991), Facultad de Ciencias de la Información, Universidad de Navarra.

EGUIZÁBAL MAZA, RAÚL (1989): El análisis del mensaje publicitario, Facultad de Ciencias de la Información, Universidad Complutense de Madrid.

ESTEBAN ALVAREZ, M. DOLORES (1998): Ensayos sobre publicidad dirigida. Facultad de Ciencias Económicas y Empresariales, Universidad de Zaragoza.

FELIU GARCIA ,EMILIO (1982): Los lenguajes de la publicidad. Facultad de Filosofía y Letras, universidad de Alicante.

FERNÁNDEZ CAVIA, JOSEP (1999): El consumidor adolescente. Motivacions, actituds i comportaments dels adolescents davant el consum, les marques i la publicitat. Facultad de Ciencias de la Comunicación, Universidad Autónoma de Barcelona.

FERRER ROSELLÓ, CLEMENTE (1991): La publicidad, una teoría humanística de su estructura, método y técnica, Facultad de Ciencias de la Información, Universidad Complutense de Madrid.

FERRÉS I PRATS, JOAN (1989): El tractament formal als espots publicitaris $i$ als videogrames didàctics: Anàlisi comparativa, Bellaterra: Publicacions de la Universidad Autònoma de Barcelona.

FLORES LÓPEZ, ENRIQUE (1999): Determinantes del grado de estandarización publicitaria, aplicación a las empresas exportadoras españolas, Facultad de Ciencias económicas y Empresariales, Universidad de Murcia..

FUEYO GUTIÉRREZ, AQUILINA (2000): Imágenes publicitarias y representaciones sociales sobre el sur. Implicaciones en la educación para el desarrollo. Facultad de Ciencias de la Educación, Universidad de Oviedo.

GALLO MARTÍNEZ, ROSALÍA (1999): Políticas de publicidad y promoción en el sector de alimentación y bebidas en España. Diferencias entre empresas familiares y no familiares, Facultad de Ingenieros Industriales, Universidad Politécnica de Cataluña. GARCIA FERNANDEZ, ROMAN (1998): Una teoria de la imagen y la publicidad en Platón. Facultad de Filosofía, Universidad de Oviedo.

GARCIA GARCIA, CARMEN (1994): La atención en la publicidad impresa: Efectos de la implicación y de la posición de la imagen. Facultad de Psicología, Universidad Autónoma de Madrid. 
GARCIA GARCIA, JOSE DIEGO (1994): Publicidad y educación: Un nuevo marco para la intervención y evaluación educativa. Facultad de Educación, Universidad de Salamanca.

GARCÍA LÓPEZ, MARCIAL (1998): Publicidad institucional: El Estado anunciante, Facultad de Ciencias de la Información, Universidad Complutense de Madrid. GARCÍA NIETO, MARÍA TERESA (1993): Acciones de relaciones públicas: La fundación, Facultad de Ciencias de la Información, Universidad Complutense de Madrid.

GARCÍA-NIETO SERRANO, FEDERICO (1975): Las técnicas audiovisuales en su aplicación a las relaciones públicas, Facultad de Ciencias Económicas y Empresariales, Universidad Complutense de Madrid.

GARCÍA RUÍZ, ALICIA (2001): Análisis de la publicidad turística española (19851999): La construcción de una imagen, Facultad de Ciencias Políticas y Sociología, Universidad de Granada.

GARCÍA-RIPOLL MONTIJANO, MARÍA ISABEL (1990): Estudio para la planificación y creación de una empresa de diseño gráfico por ordenador en el área de empresas de publicidad, diseño y artes gráficas, Escuela Técnica Superior de Ingeniería del Diseño, Universidad Politécnica de Valencia.

GARRIDO ARILLA, M. ROSA (1982): Funciones comunicacionales de la mujer en la publicidad dirigida al hombre. Análisis en tres revistas: Blanco y Negro, La Actualidad Española y Gaceta Ilustrada (1959-1979), Facultad de Ciencias de la Información, Universidad Complutense de Madrid.

GIL ALBAROVA, AMAYA (1999): Solidaridad versus competitividad en la comunicación persuasiva. Facultad de Ciencias Económicas y Empresariales, Universidad de Zaragoza.

GIQUEL ARRIBAS, OFELIA (2000): El planificador estratégico en las agencias de publicidad, Facultad de Ciencias de la Información, Universidad Complutense de Madrid.

GOMARIZ MOLINA, MANUELA (1998): Publicidad farmacéutica en El Defensor de Granada (1880-1936), Facultad de Farmacia, Universidad de Granada.

GONZALEZ CACHINERO, M. TERESA (1984): La lengua de la publicidad en la prensa. Facultad de Filosofia y Letras, Universidad de Granada.

GONZÁLEZ DÍAZ, ELSA (2001): Cadena COPE (1981-1992): Proceso de transformación en una radio competitiva, Facultad de Humanidades y Ciencias de la Comunicación, Universidad San Pablo-CEU.

GONZÁLEZ HERRANZ, M. LUISA (2001): La comunicación publicitaria en el entorno de protección al consumidor, Facultad de Ciencias de la Información, Universidad Complutense de Madrid.

GONZALEZ PESCADOR ANA M. (1998): Estrategia directiva: Análisis de eficacia y eficiencia mediante modelos de ecuaciones funcionales. Facultad de Ciencias Económicas y Empresariales, Universidad de Cantabria.

GOZÁLVEZ SERRANO, FRANCISCO (1985): Las empresas de publicidad, Facultad de Ciencias de la Información, Universidad Autónoma de Barcelona. 
GUTIÉRREZ ARRANZ, ANA (1994): La influencia de la publicidad sobre el consumidor: El efecto moderador del grado de implicación y de la estrategia publicitaria, Facultad de Ciencias Económicas y Empresariales, Universidad de Valladolid.

GUTIÉRREZ GONZÁLEZ, PEDRO PABLO (2000): Fundamentos para la gestión y dirección de ventas en publicidad. La empresa publicitaria y la actividad comercial ante un nuevo marco relacional, Facultad de Ciencias de la Información, Universidad Complutense de Madrid.

GUTIERREZ SAN MIGUEL, BEGOÑA (1992): La publicidad y el cine español en la década de los ochenta. Facultad de Geografía e Historia, Universidad de Oviedo.

HERAS PEDROSA, CARLOS DE LAS (1999): Análisis histórico de la gestión publicitaria en la prensa del movimiento en España. 1936-1984. Facultad de Ciencias de la Información, Universidad de Málaga.

HERNÁNDEZ LONGAS, ESTHER (1996): El léxico francés del automóvil a través de la publicidad, Facultad de Filología, Universidad Complutense de Madrid.

HERNÁNDEZ MARTÍNEZ, CARIDAD (1995): Recepción, contexto y creación del mensaje publicitario, Facultad de Ciencias de la Información, Universidad Complutense de Madrid.

HERNANDEZ MARTINEZ JOSE M. (1986): La publicidad en el sector agrario. Facultad ETS Ingenieros Agrónomos, Universidad Politécnica de Madrid.

HERRERO OLAIZOLA, J. (1993): Construcción del relato y valores sociales en la publicidad bancaria española, Facultad de Ciencias Políticas y Sociología, Universidad Complutense de Madrid.

HIERRO RINCON, M. LUZ (1996): Los niños y niñas ante la elección de los juguetes. Facultad de Psicología, Universidad Complutense de Madrid.

IGLESIA VILLASOL, COVADONGA (1992): Los determinantes del volumen de gastos en publicidad. Una aplicación al caso español, Facultad de Ciencias Económicas y Empresariales, Universidad Complutense de Madrid.

KIM, DOKYOON (1999): El confucionismo y el mundo de la publicidad, Facultad de Ciencias de la Información, Universidad Complutense de Madrid.

KLIATCHKO GAMALINDA, JEROME (2001): Integrated marketing communications theory and practice: The case of the Philippines. Facultad de Comunicación, Universidad de Navarra.

LANAO BERNAD, CARMEN (1992): Aproximación al estudio del lenguaje publicitario en inglés: Aspectos semánticos y pragmáticos. Facultad de Filosofía y Letras, Universidad de Zaragoza.

LAZOVSKI PIEKAREWICZ, FABIÁN (2001): Creatividad publicitaria en Uruguay. Facultad de Ciencias de la Información, Universidad Complutense de Madrid.

LEAL LÓPEZ, FRANCISCO JAVIER (1992): La publicidad. Influencias del factor legal en su gestión, Facultad de Ciencias Económicas y Empresariales, Universidad de Sevilla.

LEÓN SÁEZ YBARRA, JOSE LUIS (1985): Áreas de conocimiento y métodos cuantitativos en publicidad. Revisión crítica y estado de la cuestión, Facultad de Ciencias de la Información, Universidad Complutense de Madrid. 
LINEROS QUINTERO, ROCÍO (1995): La ciencia del texto publicitario: Una caracterización teórico-práctica de los aspectos pragmático-lingüísticos del discurso publicitario, Facultad de Letras, Universidad de Murcia.

LOMAS GARCÍA, CARLOS (1995): Pragmática de la comunicación publicitaria, Facultad de Filología, Universidad de Oviedo.

LOPEZ GUERRA, LUIS (1975): Las campañas electorales. Su regulación, evolución y características en el mundo occidental. Facultad de Derecho, Universidad Complutense de Madrid.

LÓPEZ GÓMEZ, ANTIA (1997): La dimensión de lo axiológico en el discurso del spot publicitario, Facultad de Ciencias de la Información, Universidad Complutense de Madrid.

LÓPEZ VAZQUEZ, BELÉN (2001): La comprensión psicosocial de la publicidad como técnica proyectiva. Estudio de una alternativa metodológica. Facultad de Ciencias de la Comunicación, Universidad del País Vasco.

MADRENAS I BOADAS, CARME (1987): Publicidad del empresario y coste social de la información: Publicidad y competencia, regulación de la publicidad comercial y regulación del mercado, Facultad de Derecho, Universidad Autónoma de Barcelona.

MAGALLÓN PENDÓN, SARA (1998): Relaciones públicas: Formación y profesión. Definición empírico-operativa del practicum de los estudiantes universitarios de relaciones públicas y su relación con la realidad profesional en Catalunya (1992-97), Facultad de Ciencias de la Comunicación, Universidad Autónoma de Barcelona.

MARCH PUJOL, M. ANTONIA (1995): Estudio del desarrollo del mercado de las especialidades de consejo farmacéutico y publicitarias en España y su comparación con el mercado exterior. Facultad de Ingenieros Industriales, Universidad Politécnica de Cataluña.

MARCOS MOLANO, MARÍA MAR (1995): Estética de la fotografia publicitaria en España (1975-1995), Facultad de Ciencias de la Información, Universidad Complutense de Madrid.

MARIEL CHLADKOVA, PETR (1998): Publicidad, competencia y comportamiento de mercado. Facultad de Ciencias Económicas y Empresariales, Universidad del País Vasco.

MARTÍN ARMARIO, ENRIQUE (1976): Planificación y control de campañas publicitarias, Facultad de Ciencias Económicas y Empresariales, Universidad de Sevilla.

MARTÍN GARCÍA, MARÍA DEL LIRIO (2000): La publicidad engañosa: Efectos contractuales, Facultad de Derecho, Universidad Complutense de Madrid.

MARTÍN MARTÍN, FERNANDO (1985): La Documentación publicitaria: Modelo de centro de documentación, Facultad de Ciencias de la Información, Universidad Complutense de Madrid.

MARTÍN SENTANA, JOSEFA (1996): Diseño y validación de un instrumento de medición de la eficacia publicitaria en los medios de comunicación impresos, Facultad de Ciencias Económicas y Empresariales, Universidad de Las Palmas. 
MAZO DEL CASTILLO, JUAN MANUEL (1991): Las decisiones sobre medios en comunicación por objetivos, Facultad de Ciencias de la Información, Universidad Complutense de Madrid.

MIQUEL ROMERO, M. JOSÉ (1996): Eficacia publicitaria: Repetición, frecuencia y exposición, Facultad de Ciencias Económicas y Empresariales, Universidad de Valencia.

MOHSEN ISA, WIDAD (1988): La inversión publicitaria como factor ponderante del interés de la publicidad dentro del marketing actual y aspectos diferenciativos entre publicidad sobre bienes de consumo e industriales, Facultad de Ciencias Económicas y Empresariales, Universidad de Málaga.

MONTES RODRIGUEZ, M. PILAR (1998): La oferta publicitaria como contenido contractual exigible por los consumidores y usuarios. Facultad de Derecho, Universidad de Valencia.

MORAGA GONZALEZ, JOSE LUIS (1998): Un análisis de los efectos de la publicidad y de los estandares de calidad medioambiental mínimos en modelos de competencia imperfecta. Facultad de Ciencias Sociales y Jurídicas, Universidad Carlos III de Madrid.

MORALES NAVARRO, CEFERINO (1991): La regulación normativa en la emisión de publicidad por televisión española, Facultad de Derecho, Universidad Complutense de Madrid.

MUELA MOLINA, CLARA (1996): La publicidad radiofónica en España (19241995), Facultad de Ciencias de la Información, Universidad Complutense de Madrid.

MÚJICA ALBERDI, ALAZNE (1998): Sentimiento de consumidor hacia la publicidad. Una revisión crítica de su dimensionalidad y propuesta de una escala global de medición, Facultad de Ciencias Económicas y Empresariales, Universidad de Deusto.

NOGUERO GRAU, ANTONI (1990): Concepto, desarrollo y función social de las relaciones públicas: Perspectiva histórica, teórica y jurídica, Facultad de Ciencias de la Información, Universidad Autónoma de Barcelona.

NUÑEZ GÓMEZ, PATRICIA (1992): La socialización del niño a través de la publicidad televisiva, Facultad de Ciencias de la Información, Universidad Complutense de Madrid.

OKAZAKI, SHINTARO (2001): La estandarización publicitaria de las multinacionales japonesas: Análisis de contenido de la publicidad web en Japón, España y Estados Unidos, Facultad de Ciencias Económicas y Empresariales, Universidad de Navarra.

OLARTE PASCUAL, MARIA CRISTINA (1995): La función del marketing en la organización como factor crítico de los procesos de intercambio. El caso de la prensa. Facultad de Ciencias Económicas y Empresariales, Universidad Complutense de Madrid.

OLIVARES DELGADO, FERNANDO (2001): Publicidad y ecología verde en España (1980-1999), Facultad de Ciencias Económicas y Empresariales, Universidad de Alicante. 
ORDÓÑEZ DE HARO, JOSÉ MANUEL (1999): Retailing Strategies, Facultad de Ciencias Económicas y Empresariales, Universidad Autónoma de Barcelona.

PACHECO RUEDA, MARTA (1999): Proyección y posibilidades de la publicidad exterior en España: Una propuesta de desarrollo, Facultad de Ciencias de la Información, Universidad Complutense de Madrid.

PAJUELO DE ARCOS, CARLOS (1992): Factores de éxito y fracaso en el lanzamiento y sostenimiento de una campaña publicitaria, Facultad de Ciencias de la Información, Universidad Complutense de Madrid.

PEÑA TIMÓN, VICENTE (1995): El programa narrativo como expresión del valor constitutivo del relato en el spot publicitario, Facultad de Ciencias de la Información, Universidad Complutense de Madrid.

PÉREZ BIANCO, WILFREDO (1997): Producción de comerciales para la televisión en Venezuela. Origen, proceso actual y tendencias, Facultad de Ciencias de la Comunicación, Universidad Autónoma de Barcelona.

PEREZ GAULI, JUAN CARLOS (1998): Relaciones entre arte y publicidad: La representación de la figura humana. Facultad de Ciencias de la Información, Universidad Complutense de Madrid.

PÉREZ GONZÁLEZ, RAFAEL ALBERTO (1987): Un modelo estratégico de comunicación municipal, Facultad de Ciencias de la Información, Universidad Complutense de Madrid.

PÉREZ LATRE, FRANCISCO (1993): Centrales de compra de medios, Facultad de Ciencias de la Información, Universidad de Navarra.

PÉREZ RUÍZ, MIGUEL ÁNGEL (1985): Funciones comunicativo-persuasivas del rostro en la comunicación publicitaria, Facultad de Ciencias de la Información, Universidad Complutense de Madrid.

PÉREZ TORNERO, JOSÉ MANUEL (1981): Semiótica de la persuasión publicitaria, Facultad de Ciencias de la Información, Universidad Autónoma de Barcelona.

PIÑEIRO MACEIRAS, MARÍA LUISA (1994): La predicación del discurso publicitario, Facultad de Filología, Universidad Complutense de Madrid.

PUCHOL MORENO, LUIS (1984): El discurso publicitario televisual. Facultad de Filología, Universidad de Valencia.

RAMIRO CASTELLBLANQUE, MARIANO (1996): El proceso creativo de la redacción de textos en la comunicación publicitaria, Facultad de Ciencias de la Información, Universidad Autónoma de Barcelona.

RAMOS BOSCH, TATIANA TRINIDAD (1998): Los cambios del discurso publicitario a partir de la crisis bancaria de Venezuela (1994-1997), Facultad de Ciencias de la Información, Universidad de La Laguna.

REDONDO BELLÓN, IGNACIO (1995): El alcance y la distribución de contactos publicitarios, Facultad de Ciencias Económicas y Empresariales, Universidad Autónoma de Madrid.

REY FUENTES, JUAN (1993): Los modelos masculinos en la publicidad impresa, Facultad de Ciencias de la Información, Universidad de Sevilla.

RICARTE BESCÓS, JOSÉ (1991): Una introducción específica al estudio y análisis de la creatividad, considerada como producto especifico de la comunicación 
publicitaria, Facultad de Ciencias de la Información, Universidad Autónoma de Barcelona.

RÍO PÉREZ, JORGE DEL (2001) : El entorno de trabajo en la agencia publicitaria y su influencia en la producción de la creatividad, Facultad de Ciencias de la Comunicación, Universidad de Navarra.

RIVERO SÁNCHEZ, JOSÉ ANTONIO (1991): La productora de cine publicitario. Estructura técnica, organizativa y empresarial en la industria de la producción española, Facultad de Ciencias de la Información, Universidad Autónoma de Barcelona.

ROCA CORREA, DAVID (2000): La autoedición en el departamento creativo de la agencia de publicidad: Repercusiones en el Director de arte, Facultad de Ciencias de la Comunicación, Universidad Autónoma de Barcelona.

RODRIGUEZ CARBALLEIRA, ALVARO (1991): Psicología de la persuasión coercitiva. Facultad de Psicología, Universidad de Barcelona.

RODRÍGUEZ CENTENO, JUAN CARLOS (2001): La guerra civil y el nuevo Estado: Una visión a través de la publicidad en el diario ABC de Sevilla, Facultad de Ciencias de la Información, Universidad de Sevilla.

RODRÍGUEZ MOLINA, MIGUEL A. (2001): La eficacia de las celebridades en la publicidad: Un análisis de sus variables moderadoras, Facultad de Ciencias Económicas y Empresariales, Universidad de Granada.

RODRÍGUEZ RODRÍGUEZ, JOSÉ LUIS (1992): Las motivaciones humanas en la publicidad, Facultad de Ciencias de la Información, Universidad Complutense de Madrid.

RODRÍGUEZ SOTO, ADELIZA (1996): La representación de la mujer y del hombre en la revista femenina puertorriqueña Imagen (1994-1995), Facultad de Ciencias de la Información, Universidad La Laguna.

ROYO VELA, MARCELO (1995): El contenido informativo de la publicidad y su efecto sobre la actitud hacia la publicidad y los anuncios. Un análisis empírico, Facultad de Ciencias Económicas y Empresariales, Universidad de Valencia.

SABATÉ LÓPEZ, JOAN (1998): L'us de la llengua catalana a la publicitat. Procés históric i situació actual Catalunya, Facultad de Ciencias de la Comunicación, Universidad Ramón Llull.

SABORIT VIGUER, JOSE (1986): La imagen publicitaria en televisión aproximación a cinco géneros. Facultad de Bellas Artes, Universidad Politécnica de Valencia.

SACO VÁZQUEZ, MANUELA (1995): Metodología y análisis de la imagen como factor de marketing en las entidades públicas e instituciones no lucrativas. Facultad de Ciencias Económicas u Empresariales, Universidad Complutense de Madrid.

SÁDABA CHALEZQUER, ROSARIO (1998): Comunicación comercial interactiva. El caso de la World Wide Web, Facultad de Ciencias de la Comunicación, Universidad de Navarra.

SÁEZ DÍEZ, ALFREDO (1996): Ordenación de la legislación publicitaria a partir de la ley de la publicidad, Facultad de Ciencias de la Información, Universidad de Sevilla. 
SÁNCHEZ REDONDO, MARIA ISABEL (1999): La radiodifusión católica en España. Orígenes y evolución de la Cadena de Ondas Populares Española (COPE) (1950-1980). Facultad de Humanidades y Ciencias de la Comunicación, Universidad San Pablo-CEU.

SALAS NESTARES, ISABEL (1997): La comunicación publicitaria en medios informativos interactivos: Diseño de un modelo de planificación estratégica de comunicación publicitaria interactiva, Facultad de Ciencias de la Información, Universidad Complutense de Madrid.

SÁNCHEZ CORRAL, LUIS (1989): Análisis del discurso publicitario, Facultad de Filosofía y Letras, Universidad de Málaga.

SÁNCHEZ FRANCO, MANUEL JESÚS (1997): Las variables fisicas del anuncio publicitario y su influencia en el logro de los objetivos comunicativos de marketing: Un análisis desde el procesamiento de la información publicitaria, Facultad de Ciencias Económicas y Empresariales, Universidad de Sevilla.

SÁNCHEZ GARCÍA, JAVIER (1998): La actitud hacia el anuncio: El efecto moderador de la implicación y de la fuerza de los argumentos, Facultad de Ciencias Jurídicas y Económicas, Universidad Jaume I de Castellón.

SÁNCHEZ GUZMÁN, JOSÉ RAMÓN (1974): Implicaciones económicas de la publicidad: La actividad publicitaria de la empresa, Facultad de Ciencias Económicas y Empresariales, Universidad Complutense de Madrid.

SÁNCHEZ MACÍAS, J. IGNACIO (1993): Estudio sobre la decisión publicitaria en la empresa española. (Un análisis desde la economía industrial), Facultad de Derecho, Universidad de Salamanca.

SÁNCHEZ MEDRANO, MIGUEL (1989): Publicidad y alcohol, Facultad de Ciencias de la Información, Universidad Complutense de Madrid.

SÁNCHEZ PARRA, JOSÉ (2001): La publicidad denigratoria, Facultad de Derecho, Universidad Complutense de Madrid.

SÁNCHEZ PÉREZ, JOSÉ (1989): La efectividad de la comunicación publicitaria. Un método de análisis, Facultad de Ciencias Económicas y Empresariales, Universidad de La Laguna.

SANTACREU FERNÁNDEZ, OSCAR ANTONIO (2001): La música en la publicidad, Facultad de Ciencias Económicas y Empresariales, Universidad de Alicante.

SANZ DIAZ, ALFREDO (1996): Ordenación de la legislación publicitaria a partir de la ley de la publicidad. Facultad de Ciencias de la Información, Universidad de Sevilla. SICARD CORREA, ANDRÉS (1998): Life vende, la publicidad educa, los artefactos comunican. Estudios descriptivos de los anuncios publicitarios de los artefactos de comunicar publicados en la revista Life en español, Facultad de Ciencias de la Información, Universidad de La Laguna.

SIURANA ZARAGOZA, VICTOR (1981): Teoría de la función publicitaria. Facultad de Filosofía y Ciencias de la Educación, Universidad de Barcelona.

SOLER PUJALS, PERE (1988): Dinámica de grupos y publicidad. Facultad de Ciencias de la Información, Universidad Autónoma de Barcelona. 
SUTIL MARTÍN, DOLORES LUCÍA (1991): Mensajes subliminales en la publicidad, Facultad de Psicología, Universidad Complutense de Madrid.

TAVARES DE CARVALHO LIMA, FILHO DIRCEU (1997): Programa informático para estudiantes universitarios de publicidad (estructuración de guiones televisivos). Facultad de Ciencias de la Comunicación, Universidad Autónoma de Barcelona. TORÁN TORRES, FRANCISCO EMILIO (1986): Fundamentos del posicionamiento $y$ sus implicaciones en el management del marketing estratégico, el management del producto y la estrategia publicitaria, Facultad de Ciencias Económicas y Empresariales, Universidad de Valencia.

TUDURI BORRAS, CARLES (1998): Turismo, imagen promocional y comunicación. El caso particular de las imágenes de mallorca en la prensa británica. Facultad de Ciencias de la Comunicación, Universidad Autónoma de Barcelona.

TUR VIÑES, VICTORIA (2001): Comunicación publicitaria de juguetes en televisión. Estudio de las variables audiovisuales, operaciones retóricas y aspectos creativos en una muestra de spots emitidos durante 1999 en España. Particularidades de la edad target y sexo target, Facultad de Ciencias Económicas y Empresariales, Universidad de Alicante.

VALDÉS RODRÍGUEZ, CRISTINA (1999): La traducción publicitaria como acto de comunicación intercultural. Facultad de Filología, Universidad de Oviedo.

VALLINA VELILLA, MANUEL DE LA (1981): Aplicación de la dinámica de sistemas a la modelización de ámbitos publicitarios, Facultad de Ciencias de la Información, Universidad Complutense de Madrid.

VALLS GIMENEZ, JOSEP FRANCESC (1989): Imatge de marca de pais. Facultad de Ciencias de la Información, Universidad Autónoma de Barcelona.

VAZQUEZ DIAZ, JOSE ANTONIO (1993): Análisis de diversos factores que influyen en la utilización de medicamentos: Publicidad, visita medica, prensa escrita. Facultad de Medicina, Universidad de Sevilla.

VÁZQUEZ GESTAL, MONTSERRAT MARÍA (2000): La protección jurídica de la creatividad publicitaria en los medios impresos, Facultad de Ciencias de la Información, Universidad Complutense de Madrid.

VEGA PINDADO, EUGENIO (1999): Identidad corporativa en televisión. Significación y diversidad en la gráfica televisiva. Facultad de Bellas Artes, Universidad Complutense de Madrid.

VICENTE SERRANO, PILAR (1998): Mito y publicidad: Aproximación a los mitos y símbolos de la actualidad que transmite el mensaje, Facultad de Ciencias Económicas y Empresariales, Universidad de Zaragoza.

VICTORIA MAS, JUAN SALVADOR (1998): Presencia, comunicación y estrategia de las marcas comerciales en la ficción audiovisual (product placement o emplazamiento de producto), Facultad de Ciencias de la Información, Universidad de Málaga.

VIDAL PORTABALES. JOSE IGNACIO (1997): El contrato de patrocinio publicitario en el derecho español. Facultad de Derecho, Universidad de Santiago de Compostela. 
VIRGILI RODRÍGUEZ, ANTONIO (1998): Gestión publicitaria en la empresa de radio, Facultad de Ciencias de la Comunicación, Universidad de Navarra.

VISIEDO CLAVEDOL, ROSA (1997): El futuro de las agencias de publicidad en el marco de la Comunidad valenciana: una investigación aplicada, Facultad de Ciencias de la Información, Universidad Complutense de Madrid.

ZALDUMBIDE AMEZAGA, IÑAKI (1996): Identidad visual corporativa: La teoría y la práctica. Estudio de la creación de la nueva identidad visual de las Cajas vascas, Facultad de Ciencias Sociales y de la Comunicación, Universidad del País Vasco.

\section{BIBLIOGRAFÍA}

AGUDELO, DIANA; BRETÓN-LÓPEZ, JUANA; ORTÍZ-RECIO, GINÉS; POVEDA-VERA, JORGE; TEVA, INMACULADA (2003); "Análisis de la productividad científica de la psicología científica a través de las tesis doctorales", Psicothema, 15 (4): 595-609.

BOJO CANALES, C.; CARABANTES ALARCÓN, D.; VEIGA DE CABO, J.; MARTÍNEZ HERNÁNDEZ, D. (2004): “Análisis bibliométrico de la Revista Española de Quimioterapia (1996-2000)", Rev Esp Quimioterap, 17 (2): 161-168.

CASTERA, V.T.; SANZ VALERO, J.; JUAN-QUILIS, V.; WANDEN-BERGHE, C.; CULEBRAS, J.M.; GARCÍA DE LORENZO, A. (2008): "Estudio bibliométrico de la revista Nutrición Hospitalaria en el período 2001 a 2005: Parte II, análisis de la producción científica", Nutrición Hospitalaria, 23 (5): 469-476.

CASTERA, V.T.; SANZ VALERO, J.; JUAN-QUILIS, V.; WANDEN-BERGHE, C.; CULEBRAS, J.M.; GARCÍA DE LORENZO, A. (2008): "Estudio bibliométrico de la revista Nutrición Hospitalaria en el período 2001 a 2005: Parte 2, análisis de consumo; las referencias bibliográficas", Nutrición Hospitalaria, 23 (6): 541-546.

CEOLIN POLETTO, V.; MEDRIROS FARACO, I. (2010): "Bibliometric study of articles published in a Brazilian journal of pediatric dentistry", Braz Oral Res., 24 (1): 83-88.

CONSEJO DE UNIVERSIDADES, SECRETARÍA GENERAL (1990): Tesis Doctorales 1976-77 / 1988-89, Madrid: Consejo de Universidades, 3 vols.

DELGADO LÓPEZ-CÓZAR, E.; TORRES SALINAS, D.; JIMÉNEZ CONTRERAS, E.; RUÍZ PÉREZ, R. (2006): “Análisis bibliométrico y de redes sociales aplicado a las tesis bibliométricas defendidas en España (1976-2002): Temas, escuelas científicas y redes académicas", Revista Española de Documentación Científica, 29 (4): 493-524.

FACULTAD DE CIENCIAS DE LA INFORMACIÓN (1983): "Tesis Doctorales (1978-1982)", en Bibliografía de las Ciencias de la Información, Madrid: Facultad de Ciencias de la Información, Universidad Complutense de Madrid, pp. 86-87.

FINKENSTAEDT, T. (1990): "Measuring research performance in the Humanities", Scientometrics, 19, 409-417. 
FUENTES PUJOL, EULALIA; ARGUIMBAU VIVÓ, LLORENÇ (2010): "Las tesis doctorales en España (1997-2008): Análisis, estadísticas y repositorios cooperativos", Revista Española de Documentación Científica, 33 (1): 63-89.

GARFIELD, EUGENE (2003): "The meaning of the impact factor", International Journal of Clinical and Health Psychology, 3 (2): 363-369.

GLÄNZEL, WOLFGANG (2003): Bibliometrics As a Research Field (A Course on Theory and Application of Bibliometric Indicators), Leuven: Katholieke Universiteit Leuven. Course Handouts.

GODIN, BENOIT (2006): On The Origins of Bibliometrics, Quebec: Project on The History and Sociology of S \& T Statistics, Working Paper No. 33.

GÓMEZ CARIDAD, I.; FERNÁNDEZ MUÑOZ, M.T.; BORDONS GANGAS, M.; MORILLO ARIZA, F. (2004): "La producción científica española en medicina en los años 1994-1999”, Revista Clínica Española, 204 (2): 75-88.

HERNÁNDEZ VAQUERO, D.; FERNÁNDEZ FERNÁNDEZ, J.M.; GARCÍA SANDOVAL, M.A. (1995): "La producción científica española en cirugía ortopédica y traumatología", Rev Esp Cir Osteoart, 30: 123-129.

HUBER, JOHN C., WAGNER-DÖBLER, ROLAND (2001): "Scientific production: A statistical analysis of authors in mathematical logic", Scientometrics, 50 (2): 323337.

JONES, DANIEL E. (1998): "Investigación sobre comunicación en España. Evolución y perspectivas", Zer, 5: 13-51.

JONES, DANIEL E.; BARÓ I QUERALT, JAUME (1997): “Tesis doctorals i traballs de recerca universitaris sobre comunicació als països Catalans, 1954-1996: Aproximació bibliomètrica", Anàlisis, 20: 157-187.

KLAIC, B. (1995): "Analysis of the scientific productivity of researchers from the Republic of Croatia for the period 1990-1992", Scientometrics, 32 (2): 133-152.

KRAUSDKOPF, M. (1994): "Epistemometria, a term contributing to express the meaning and potential methodologies of scientometrics in spanish speaking countries", Scientometrics, 30 (2-3): 425-428.

LAZAREV, V.S. (1997): "Properties of scientific periodicals under bibliometric assessment", International Journal of Information Sciences for Decision Making, 1: $1-17$.

LEÓN SÁEZ DE IBARRA, JOSÉ LUIS (1988): La investigación en publicidad: Metodología y crítica, Universidad del País Vasco, Servicio Editorial.

LÓPEZ LÓPEZ, PEDRO (1996): "La investigación bibliométrica en España (tesis doctorales)", Revista Española de Documentación Científica, 19 (1): 84-89.

LÓPEZ LÓPEZ, PEDRO (1996): Introducción a la bibliometría, Valencia: Editorial Promolibro.

LÓPEZ PIÑERO, J.M.; TERRADA, M.L. (1992): "Los indicadores bibliométricos y la evaluación de la actividad médico-científica. (I) Uso y abusos de la bibliometría", Med Clin (Barc), 98: 64-68.

LÓPEZ PIÑERO, J.M.; TERRADA, M.L. (1992): "Los indicadores bibliométricos y la evaluación de la actividad médico-científica. (II) La comunicación científica en las distitntas áreas de las ciencias sociales", Med Clin (Barc), 98: 101-106. 
LÓPEZ PIÑERO, J.M.; TERRADA, M.L. (1992): "Los indicadores bibliométricos y la evaluación de la actividad médico-científica. (III) Los indicadores de producción, circulación y dispersión, consumo de información y repercusión", Med Clin (Barc), 98: 142-148.

LÓPEZ PIÑERO, J.M.; TERRADA, M.L. (1992): "Los indicadores bibliométricos y la evaluación de la actividad médico-científica. (IV) La aplicación de los indicadores", Med Clin (Barc), 98: 384-388.

LÓPEZ YEPES, J.; ROS, J.; ORERA, L.; FERNÁNDEZ, M.T.; PRAT, J. (2005): Las tesis doctorales. Producción, evaluación y defensa, Madrid: Fragua.

LÓPEZ YEPES, JOSÉ (2002): "Focos de investigación y escuelas científicas en Documentación: La experiencia de las tesis doctorales", El profesional de la información, 1 (11): 46-51.

LÓPEZ YEPES, JOSÉ (2002): “Focos de investigación y escuelas científicas en Documentación a través de la dirección y realización de tesis doctorales: El caso del Departamento de Biblioteconomía y Documentación de la Universidad Complutense de Madrid (1983-2001)", Documentación de las ciencias de la información, 25: 19-54.

MARTÍN MARTÍN, FERNANDO (1983): Las bases de datos publicitarias: Estudio bibliográfico de la comunicación publicitaria española (1900-1983), Madrid: Duplo, S.A. y Departamento de Documentación de la Facultad de Ciencias de la Información, Universidad Complutense de Madrid.

MARTÍNEZ PESTAÑA, MARÍA JESÚS (2004): "La producción de tesis doctorales sobre temas publicitarios", Documentación de las Ciencias de la Información, 27: 237-267.

MÉNDIZ NOGUERO, ALFONSO (2000): “Orígenes, evolución y desafíos actuales de la docencia publicitaria en España”, Comunicación y Sociedad, XIII (2): 181-225.

MICHALOPOULOS, ARGYRIS; FALAGAS, M.E. (2005): "A bibliometric analysis of global research production in respiratory medicine", Chest, 128: 3993-3998.

MILANÉS GUISADO, Y.; PÉREZ RODRÍGUEZ, Y.; PERALTA GONZÁLEZ, J.; RUÍZZ RAMOS, M.E. (2008): "Los estudios de evaluación de la ciencia: Aproximación teórico-métrica", ACIMED, 18 (6): 1-28.

NEDERHOF, A.J.; VAN RAAN, R.A.; DE BRUIN, R.E.; DEKKER, P.J. (1989): "Assessing the usefulness of bibliometric indicators for the humanities and the social behavioral sciences: A comparative study", Scientometrics, 15, 423-435.

OSAREH, FARIDEH (2003): "The use and application of multivariate analysis techniques in bibliometric and scientometric studies", Iranian Journal of Information Science \& Technology, 1 (2): 59-71.

PALOMAR, T.; GARCIA-HERAS, M.; VILLEGAS, M.A. (2009): “Archaelogical and historical glasses: A bibliometric study", Boletín de la Sociedad Española de Cerámica y Vidrio, 48 (4): 187-194.

ROSTAING, HERVÉ; BARTS, N.; LÉVSILLÉ, V. (2007): "Bibliometrics: Representation instrument of the multidisciplinary positioning of a scientific area. Implementation for and advisor scientific committee", La interdisciplinariedad y la 
transdisciplinariedad en la organización del conocimiento científico (Actas del VIII Congreso ISKO), León: Congreso ISKO.

SÁNCHEZ ESPINOSA, JUAN FRANCISCO (2009): Análisis de la producción científica sobre el manejo terapéutico del paciente asmático (1955-2005), Murcia: Universidad de Murcia. Tesis doctoral.

SÁNCHEZ GUZMÁN, JOSÉ RAMÓN (1989): Breve historia de la publicidad, Madrid: Editoral Ciencia 3, S.A.

SÁNCHEZ GUZMÁN, JOSÉ RAMÓN (1980): "El tratamiento científico de la publicidad", Publitecnia, 54: 125-143.

SÁNCHEZ REVILLA, MIGUEL ÁNGEL (1998): "La inversión publicitaria en los medios: De los grandes crecimientos de la transición y la década de los 80 , a la crisis y madurez de finales del siglo", Ip Mark, 511: 19-22.

TARRÉS, M. CRISTINA (2009): "Bibliometric analysis of the Journal of Medicine and Movies (2005-2008)", Journal of Medicine and Movies, 5: 106-115.

VAN LEEUWEN, THED (2006): "The application of bibliometric analyses in the evaluation of social science research. Who benefits from it, and why it is still feasible", Scientometrics, 66 (1): 133-154.

VILLAGRÁ RUBIO, A. (1992): "Scientific production of Spanish universities in the fields of social sciences and language", Scientometrics, 24 (1): 3-19.

WEN-TA, CHIU; JING-SHAN, HUANG; YUH-SHAN, HO (2004): "Bibliometric analysis of Severe Acute Respiratory Syndrome - related research in the beginning stage", Scientometrics, 61 (1): 69-77.

YANG, LIN (1996): "Empirical studies of negative political advertising: A quantitive review using a method of combinec citation and context analysis", Scientometrics, 37 (3): 385-399.

ZULUETA, MARÍA ÁNGELES (2002): "Bibliometría y métodos bibliométricos", en J. López Yepes (Coord.): Manual de Ciencias de la Documentación, Madrid: Ediciones Pirámide; pp. 117-136.

ZULUETA, MARIA ÁNGELES; CABRERO, A.; BORDONS, M. (1999): "Identificación y estudio de grupos de investigación a través de indicadores bibliométricos", Rev Esp Doc Cient, 22: 333-347. 
ANEXO: Tablas

Tabla 1

Número de tesis doctorales por años y porcentaje (\%) desde 1971-2001

\begin{tabular}{|c|c|c|}
\hline Año & Número de Tesis & $\%$ \\
\hline 1974 & 1 & 0,61 \\
\hline 1975 & 2 & 1,21 \\
\hline 1976 & 1 & 0,61 \\
\hline 1979 & 1 & 0,61 \\
\hline 1981 & 4 & 2,42 \\
\hline 1982 & 2 & 1,21 \\
\hline 1984 & 2 & 1,21 \\
\hline 1985 & 4 & 2,42 \\
\hline 1986 & 3 & 1,81 \\
\hline 1987 & 2 & 1,21 \\
\hline 1988 & 2 & 1,21 \\
\hline 1989 & 7 & 4,24 \\
\hline 1990 & 2 & 1,21 \\
\hline 1991 & 8 & 4,84 \\
\hline 1992 & 7 & 4,24 \\
\hline 1993 & 10 & 6,06 \\
\hline 1994 & 7 & 4,24 \\
\hline 1995 & 11 & 6,67 \\
\hline 1996 & 11 & 6,67 \\
\hline 1997 & 10 & 6,06 \\
\hline 1998 & 22 & 13,35 \\
\hline 1999 & 16 & 9,69 \\
\hline 2000 & 10 & 6,06 \\
\hline 2001 & 20 & 12,14 \\
\hline Total & 165 & 100 \\
\hline
\end{tabular}


Tabla 2

Número de tesis doctorales por período de años (1971-2000) y 2001

Fuente: Elaboración propia

\begin{tabular}{|l|l|}
\hline Período de años & Número de tesis \\
\hline $\mathbf{1 9 7 1 - 1 9 8 0}$ & 5 \\
\hline $\mathbf{1 9 8 1 - 1 9 9 0}$ & 28 \\
\hline $\mathbf{1 9 9 1 - 2 0 0 0}$ & 112 \\
\hline $\mathbf{2 0 0 1}$ & 20 \\
\hline \multicolumn{2}{|l}{$\mathbf{1 6 5}$} \\
\hline
\end{tabular}

Tabla 3

Número total de tesis por niveles de productividad de universidades agrupadas

\begin{tabular}{|l|l|l|}
\hline Grupo & Total tesis doctorales & Número tesis acumulado \\
\hline $\mathbf{( 1 )}$ & $\mathbf{5 6}$ & 56 \\
\hline $\mathbf{( 2 )}$ & $\mathbf{3 0}$ & 86 \\
\hline $\mathbf{( 3 )}$ & $\mathbf{3 8}$ & 124 \\
\hline $\mathbf{( 4 )}$ & $\mathbf{3 1}$ & 155 \\
\hline $\mathbf{( 5 )}$ & $\mathbf{1 0}$ & $\mathbf{1 6 5}$ \\
\hline
\end{tabular}

Fuente: Elaboración propia

Tabla 4

Número de tesis doctorales por universidades

\begin{tabular}{|l|l|l|l|}
\hline & Universidad & Año (número tesis por año) & Total \\
\hline (1) & Universidad Complutense & $1974(1), 1975(2), 1981(1), 1982$ & \\
& & $(1), 1985(3), 1987(1), 1989(2)$, & \\
& & $1991(4), 1992(4), 1993(4), 1994$ & \\
& & $(2), 1995(5), 1996(3), 1997(4)$, & \\
& & $1998(2), 1999(5), 2000(6), 2001$ & \\
& & & $\mathbf{5 6}$ \\
\hline (2) & Universidad Autónoma de Barcelona & $1981(1), 1985(1), 1987(1), 1988$ & $\mathbf{1 7}$ \\
& & $(1), 1989(2), 1990(1), 1991(2)$, & \\
& & $1996(1), 1997(2), 1998(2), 1999$ & $\mathbf{1 3}$ \\
\hline
\end{tabular}




\begin{tabular}{|c|c|c|c|}
\hline & Universidad de Navarra & $\begin{array}{l}(2), 2000(1) \\
1979(1), 1989(1), 1993(2), 1994 \\
(2), 1998(3), 2001(4)\end{array}$ & \\
\hline (3) & $\begin{array}{l}\text { Universidad de Sevilla } \\
\text { Universidad de Valencia } \\
\text { Universidad de Oviedo } \\
\text { Universidad de Alicante } \\
\text { Universidad de Granada } \\
\text { Universidad de Málaga }\end{array}$ & $\begin{array}{l}1976(1), 1991(1), 1992(1), 1993 \\
(2), 1996(2), 1997(1), 2001(1) \\
1981(1), 1984(1), 1986(1), 1993 \\
(1), 1995(1), 1996(1), 1997(1), \\
1998(1) \\
1992(1), 1995(2), 1998(1), 1999 \\
(1), 2000(1) \\
1982(1), 1999(1), 2001(3) \\
1998(1), 1998(1), 1999(1), 2001 \\
(2) \\
1988(1), 1989(1), 1998(1), 1999 \\
(1), 2000(1)\end{array}$ & $\begin{array}{l}9 \\
8 \\
6 \\
5 \\
5 \\
5\end{array}$ \\
\hline (4) & $\begin{array}{l}\text { Universidad de La Laguna } \\
\text { Universidad de Zaragoza } \\
\text { Universidad del País Vasco } \\
\text { Universidad de Salamanca } \\
\text { Universidad Politécnica de Valencia } \\
\text { Universidad Autónoma de Madrid } \\
\text { Universidad Carlos III } \\
\text { Universidad de Barcelona } \\
\text { Universidad de Murcia } \\
\text { Universidad Politécnica de Cataluña } \\
\text { Universidad Ramón Llull } \\
\text { Universidad San Pablo-CEU }\end{array}$ & $\begin{array}{l}1989(1), 1998(2), 1999(1) \\
1992(1), 1998(2), 1999(1) \\
1996(1), 1998(1), 2001(1) \\
1993(1), 1994(1), 2001(1) \\
1986(1), 1990(1), 2000(1) \\
1994(1), 1995(1) \\
1997(1), 1998(1) \\
1981(1), 1991(1) \\
1995(1), 1999(1) \\
1995(1), 1999(1) \\
1998(1), 2001(1) \\
1999(1), 2001(1)\end{array}$ & $\begin{array}{l}4 \\
4 \\
3 \\
3 \\
3 \\
3 \\
2 \\
2 \\
2 \\
2 \\
2 \\
2 \\
2 \\
2 \\
2\end{array}$ \\
\hline (5) & $\begin{array}{l}\text { Universidad de Cantabria } \\
\text { Universidad de Comillas } \\
\text { Universidad de Deusto } \\
\text { Universidad Jaume I de Castellón } \\
\text { Universidad de La Laguna } \\
\text { Universidad de Las Palmas } \\
\text { Universidad de Santiago de } \\
\text { Compostela } \\
\text { Universidad de Valladolid } \\
\text { Universidad Nac. Educación a } \\
\text { Distancia } \\
\text { Universidad Politécnica de Madrid }\end{array}$ & $\begin{array}{l}1998(1) \\
1998(1) \\
1998(1) \\
1998(1) \\
1996(1) \\
1996(1) \\
1997(1) \\
1994(1) \\
1996(1) \\
1986(1)\end{array}$ & $\begin{array}{l}1 \\
1 \\
1 \\
1 \\
1 \\
1 \\
1 \\
1 \\
1 \\
1 \\
1\end{array}$ \\
\hline & 31 Universidades & Tesis doctorales & 165 \\
\hline
\end{tabular}

Fuente: Elaboración propia 


\section{Tabla 5}

Facultades donde se han defendido tesis de contenido publicitario

\begin{tabular}{|l|l|}
\hline Facultad & Total \\
\hline C. Información / Comunicación & 84 \\
\hline C. Económicas / Empresariales & 33 \\
\hline Derecho & 9 \\
\hline Filología & 8 \\
\hline Filosofía y Letras & 8 \\
\hline Psicología & 5 \\
\hline C. Políticas / Sociología & 4 \\
\hline Ingeniería / E.T.S. & 4 \\
\hline Bellas Artes & 3 \\
\hline Educación & 3 \\
\hline Medicina & 2 \\
\hline Farmacia & 1 \\
\hline Geografía e Historia & 1 \\
\hline
\end{tabular}

Fuente: Elaboración propia

\section{Tabla 6}

Facultades en que se han presentado tesis doctorales

\begin{tabular}{|c|c|c|c|}
\hline Facultad & Univ. & $\begin{array}{l}\text { Año (número de } \\
\text { tesis) }\end{array}$ & Total \\
\hline $\begin{array}{l}\text { C. Información / } \\
\text { Comunicación }\end{array}$ & $\begin{array}{l}\text { UCM } \\
\text { UAB } \\
\text { UN } \\
\text { ULL }\end{array}$ & $\begin{array}{l}1981(1), 1982(1), \\
1985(3), 1987(1), \\
1989(2), 1991(2), \\
1992(3), 1993(3), \\
1994(1), 1995(3), \\
1996(1), 1997(4), \\
1998(2), 1999(4), \\
2000(5) \\
1981(1), 1985(1), \\
1988(1), 1989(2),\end{array}$ & $\begin{array}{l}41 \\
15 \\
12 \\
4\end{array}$ \\
\hline
\end{tabular}




\begin{tabular}{|c|c|c|c|}
\hline & $\begin{array}{l}\text { US } \\
\text { UMA } \\
\text { UPV } \\
\text { URL } \\
\text { USP }\end{array}$ & $\begin{array}{l}1990(1), 1991(2), \\
1996(1), 1997(2), \\
1998(2), 1999(1), \\
2000(1) \\
1979(1), 1989(1), \\
1993(2), 1994(2), \\
1998(3), 2001(3) \\
1996(1), 1998(2), \\
1999(1) \\
1993(1), 1996(2), \\
2001(1) \\
1998(1), 1999(1) \\
1996(1), 2001(1) \\
1998(1), 2001(1) \\
1999(1), 2001(1)\end{array}$ & $\begin{array}{l}4 \\
2 \\
2 \\
2 \\
2\end{array}$ \\
\hline $\begin{array}{l}\text { C. Económicas / } \\
\text { Empresariales }\end{array}$ & $\begin{array}{l}\text { UCM } \\
\text { UA } \\
\text { UV } \\
\text { US } \\
\text { UZ } \\
\text { UG } \\
\text { UMA } \\
\text { ULL } \\
\text { UVL } \\
\text { UAM } \\
\text { ULP } \\
\text { UCA } \\
\text { UC } \\
\text { UD } \\
\text { UMU } \\
\text { UAB } \\
\text { UN } \\
\text { UPV }\end{array}$ & $\begin{array}{l}1974(1), 1975(1), \\
1992(1), 1995(2) \\
1999(1), 2001(3) \\
1986(1), 1995(1), \\
1996(1), 1997(1) \\
1976(1), 1992(1), \\
1997(1) \\
1998(2), 1999(1) \\
1999(1), 2001(1) \\
1988(1) \\
1989(1) \\
1994(1) \\
1995(1) \\
1996(1) \\
1998(1) \\
1998(1) \\
1998(1) \\
1999(1) \\
1999(1) \\
2001(1) \\
1998(1)\end{array}$ & $\begin{array}{l}5 \\
4 \\
4 \\
3 \\
3 \\
2 \\
1 \\
1 \\
1 \\
1 \\
1 \\
1 \\
1 \\
1 \\
1 \\
1 \\
1 \\
1\end{array}$ \\
\hline Derecho & $\begin{array}{l}\text { UCM } \\
\text { UAB } \\
\text { UJC } \\
\text { USA } \\
\text { USC } \\
\text { UV }\end{array}$ & $\begin{array}{l}1975(1), 1991(1), \\
2000(1), 2001(1) \\
1987(1) \\
1998(1) \\
1993(1) \\
1997(1) \\
1998(1)\end{array}$ & $\begin{array}{l}4 \\
1 \\
1 \\
1 \\
1 \\
1\end{array}$ \\
\hline Filología & $\begin{array}{l}\text { UCM } \\
\text { UO } \\
\text { UV }\end{array}$ & $\begin{array}{l}1994(1), 1996(1) \\
1995(1), 1999(1) \\
1984(1), 1993(1)\end{array}$ & $\begin{array}{l}2 \\
2 \\
2\end{array}$ \\
\hline
\end{tabular}




\begin{tabular}{|c|c|c|c|}
\hline & $\begin{array}{l}\text { UMA } \\
\text { USA }\end{array}$ & $\begin{array}{l}2000(1) \\
2001(1)\end{array}$ & $\begin{array}{l}1 \\
1\end{array}$ \\
\hline Filosofía y Letras & $\begin{array}{l}\text { UA } \\
\text { UB } \\
\text { UG } \\
\text { UMA } \\
\text { UMU } \\
\text { UO } \\
\text { UV } \\
\text { UZ }\end{array}$ & $\begin{array}{l}1982(1) \\
1981(1) \\
1984(1) \\
1989(1) \\
1995(1) \\
1998(1) \\
1981(1) \\
1992(1)\end{array}$ & $\begin{array}{l}1 \\
1 \\
1 \\
1 \\
1 \\
1 \\
1 \\
1 \\
1 \\
1\end{array}$ \\
\hline Psicología & $\begin{array}{l}\text { UCM } \\
\text { UAM } \\
\text { UB } \\
\text { UO }\end{array}$ & $\begin{array}{l}1991(1), 1996(1) \\
1994(1) \\
1991(1) \\
1995(1)\end{array}$ & $\begin{array}{l}2 \\
1 \\
1 \\
1\end{array}$ \\
\hline $\begin{array}{l}\text { C. Políticas / } \\
\text { Sociología }\end{array}$ & $\begin{array}{l}\text { UCIII } \\
\text { UCM } \\
\text { UG }\end{array}$ & $\begin{array}{l}1997(1), 1998) \\
1993(1) \\
2001(1)\end{array}$ & $\begin{array}{l}2 \\
1 \\
1\end{array}$ \\
\hline Ingeniería / E.T.S. & $\begin{array}{l}\text { UPC } \\
\text { UPM } \\
\text { UPVA }\end{array}$ & $\begin{array}{l}1995(1), 1999(1) \\
1986(1) \\
1990(1)\end{array}$ & $\begin{array}{l}2 \\
1 \\
1\end{array}$ \\
\hline Bellas Artes & $\begin{array}{l}\text { UPVA } \\
\text { UCM }\end{array}$ & $\begin{array}{l}1986(1), 2000(1) \\
1999(1)\end{array}$ & $\begin{array}{l}2 \\
1\end{array}$ \\
\hline Educación & $\begin{array}{l}\text { UNED } \\
\text { USA } \\
\text { UO }\end{array}$ & $\begin{array}{l}1996(1) \\
1994(1) \\
2000(1)\end{array}$ & $\begin{array}{l}1 \\
1 \\
1\end{array}$ \\
\hline Medicina & US & $1991(1), 1993(1)$ & 2 \\
\hline Farmacia & UG & $1998(1)$ & 1 \\
\hline Geografía e Historia & UO & $1992(1)$ & 1 \\
\hline
\end{tabular}

Tabla 7

Líneas de investigación presentes en las tesis doctorales

\begin{tabular}{|l|l|c|}
\hline Línea de investigación & (Año lectura de tesis doctoral) Autor & Tesis \\
\hline & $\begin{array}{l}\text { (1984) González Cachinero; (1986) Saborit Viguer; (1989) } \\
\text { Ferrés i Prats; (1991) Rivero Sánchez; (1992) Gutiérrez San } \\
\text { Miguel; (1994) Domínguez Ávila; (1994) C. García García; } \\
\text { (1996) Muela Molina; (1997) Salas Nestares; (1998) Sádaba }\end{array}$ & \\
& $\begin{array}{l}\text { Chalezquer; (1998) Victoria Mas; (1998) Sicard Correa; } \\
\text { (1999) Alvarez Roque, (1999) Betés Rodríguez; (1999) } \\
\text { Calderón Martínez;(1999) Sánchez Redondo; (1999) Amiguet }\end{array}$ & $\mathbf{2 0}$ \\
\hline
\end{tabular}




\begin{tabular}{|c|c|c|}
\hline & $\begin{array}{l}\text { Esteban; (1999) Pacheco Rueda; (2001) Díaz Soloaga; (2001) } \\
\text { González Díaz }\end{array}$ & \\
\hline Campañas publicitarias & $\begin{array}{l}\text { (1975) López Guerra; (1989) Sánchez Medrano; (1992) } \\
\text { Pajuelo de Arcos; (1993) Herrero Olaizola; (1995) March } \\
\text { Pujol; (1996) Hernández Longas; (1996) Zaldumbide } \\
\text { Amezaga; (1998) Bocigas Solar; (1998) Gomariz Molina; } \\
\text { (1998) García López; (1999) Barrio García; (1999) Gallo } \\
\text { Martínez; (2001) Álvarez Ruíz; (2001) Rodríguez Centeno; } \\
\text { (2001) Santacreu Fernández; (2001) Tur Viñes; (2001) García } \\
\text { Ruíz. }\end{array}$ & 17 \\
\hline $\begin{array}{l}\text { Consumidor / } \\
\text { impacto de la publicidad }\end{array}$ & $\begin{array}{l}\text { (1988) Soler Pujals; (1991) Amaya Román, (1991) Rodríguez } \\
\text { Carballeira; (1992) Nuñez Gómez; (1992) Rodríguez } \\
\text { Rodríguez; (1993) Vázquez Díaz, (1994) Gutiérrez Arranz; } \\
\text { (1996) Hierro Rincón; (1998) Bringue Sala; (1998) Esteban } \\
\text { Alvarez; (1998) Sánchez García; (1998) Moraga González; } \\
\text { (1998) Mújica Alberdi; (1999) Fernández Cavia; (2001) } \\
\text { Carrillo Durán; (2001) González Herranz; (2001) López } \\
\text { Vázquez }\end{array}$ & 17 \\
\hline Mensaje & $\begin{array}{l}\text { (1982) Feliu García; (1989) Eguizábal Maza; (1989) Valls } \\
\text { Giménez; (1991) Sutil Martín; (1992) Lanao Bernad; (1993) } \\
\text { Conejero López; (1995) Díez Arroyo; (1995) Hernández } \\
\text { Martínez; (1995) Peña Timón; (1998) Vicente Serrano; (1999) } \\
\text { Valdés Rodríguez; (1999) Vega Pindado; (2000) Bohoyo } \\
\text { Díaz; (2000) Comitre Narváez; (2001) Durán Martínez }\end{array}$ & 15 \\
\hline Eficacia & $\begin{array}{l}\text { (1985) León Sáez Ybarra; (1986) Torán Torres; (1989) } \\
\text { Sánchez Pérez; (1995) Saco Vázquez; (1996) Miquel Romero; } \\
\text { (1996) Martín Sentana; (1997) Descalzo González; (1997) } \\
\text { Sánchez Franco; (1998) González Pescador; (1999) Gil } \\
\text { Albarova; (2001) Cascante Serratosa; (2001) De Mooij; } \\
\text { (2001) Kliatchko Gamalinda; (2001) Rodríguez Molina. }\end{array}$ & 14 \\
\hline Elementos simbólicos & $\begin{array}{l}\text { (1982) Garrido Arilla; (1985) Pérez Ruíz; (1993) Rey Fuentes; } \\
\text { (1994) Baca Lagos, (1996) Rodríguez Soto; (1998) García } \\
\text { Fernández; (1998) Pérez Gauli; (1998) Sabaté López; (1998) } \\
\text { Tuduri Borras; (1999) Kim; (2000) Fueyo Gutiérrez; (2001) } \\
\text { Olivares Delgado. }\end{array}$ & 12 \\
\hline Empresa de publicidad & $\begin{array}{l}\text { (1974) Sánchez Guzmán; (1985) Gozálvez Serrano; (1985) } \\
\text { Martín Martín; (1990) García-Ripoll Montijano; (1993) Arceo } \\
\text { Vacas, A.; (1993) Pérez Latre; (1993) García Nieto; (1997) } \\
\text { Pérez Bianco; (1997) Visiedo Clavedol; (2000) Giquel } \\
\text { Arribas; (2001) Río Pérez. }\end{array}$ & 11 \\
\hline
\end{tabular}




\begin{tabular}{|c|c|c|}
\hline Legislación & $\begin{array}{l}\text { (1979) Desantes Guanter; (1987) Madrenas i Boadas; (1989) } \\
\text { Corredoira Alfonso; (1991) Morales Navarro; (1996) Sáez } \\
\text { Díez; (1996) Sanz Díaz; (1997) Vidal Portabales; (1998) } \\
\text { Montes Rodríguez; (2000) Martín García; (2001) Sánchez } \\
\text { Parra. }\end{array}$ & 10 \\
\hline Gestión & $\begin{array}{l}\text { (1976) Martín Armario; (1992) Leal López; (1993) Sánchez } \\
\text { Macías; (1995) Olarte Pascual; (1998) Mariel Chladkova; } \\
\text { (1998) Virgili Rodríguez; (1999) Heras Pedrosa; (2000) } \\
\text { Blasco López; (2000) Gutiérrez González. }\end{array}$ & 9 \\
\hline Creatividad & $\begin{array}{l}\text { (1991) Ricarte Bescós; (1993) Angeles Villena; (1995) } \\
\text { Marcos Molano; (1996) Ramiro Castellblanque; (2000) Baños } \\
\text { González; (2000) Roca Correa; (2000) Vázquez Gestal; } \\
\text { (2001) Lazovski piekarevicz }\end{array}$ & 8 \\
\hline Discurso & $\begin{array}{l}\text { (1984) Puchol Moreno; (1989) Sánchez Corral; (1994) Piñeiro } \\
\text { Maceiras; (1995) Lomas García; (1995) Lineros Quintero; } \\
\text { (1996) Correa García; (1997) López Gómez; (1998) Ramos } \\
\text { Bosch. }\end{array}$ & 8 \\
\hline Planificación de medios & $\begin{array}{l}\text { (1991) Mazo del Castillo; (1995) Redondo Bellón; (1997) } \\
\text { Aldás Manzano; (1997) Díez Medrano. }\end{array}$ & 4 \\
\hline Docencia & $\begin{array}{l}\text { (1981) Beltrán de Tena; (1994) J.D. García García; (1997) } \\
\text { Tavares de Carvalho }\end{array}$ & 3 \\
\hline Función de la publicidad & $\begin{array}{l}\text { (1981) Siurana Zaragoza; (1991) Ferrer Roselló; (1995) Royo } \\
\text { Vela. }\end{array}$ & 3 \\
\hline Inversión publicitaria & $\begin{array}{l}\text { (1986) Hernández Martínez; (1988) Mohsen Isa; (1992) } \\
\text { Iglesia Villasol. }\end{array}$ & 3 \\
\hline Relaciones públicas & $\begin{array}{l}\text { (1975) García-Nieto Soriano; (1990) Noguero Grau; (1998) } \\
\text { Magallón Pendón. }\end{array}$ & 3 \\
\hline Estandarización & (1999) Flores López; (2001) Okazaki. & 2 \\
\hline Estrategia & (1987) Pérez González; (1999) Ordóñez de Haro. & 2 \\
\hline Semiótica & (1981) Pérez Tornero; (1993) Caro Almeda. & 2 \\
\hline Patrocinio & (1994) Carrogio Guerín. & 1 \\
\hline Teoría de sistemas & (1981) Vallina Velilla. & 1 \\
\hline
\end{tabular}

Fuente: Elaboración propia 


\section{Tabla 8}

Distribución de lenguas en tesis doctorales (1971-2001)

\begin{tabular}{|l|c|}
\hline Lengua & $\begin{array}{c}\text { Número } \\
\text { monografias }\end{array}$ \\
\hline Castellano & 160 \\
\hline Catalán & 3 \\
\hline Inglés & 2 \\
\hline Total tesis & $\mathbf{1 6 5}$ \\
\hline
\end{tabular}

Fuente: Elaboración propia 